\title{
Implementación de la metodología TPM-Lean Manufacturing para mejorar la eficiencia general de los equipos (OEE) en la producción de repuestos en una empresa metalmecánica
}

Nohemy Canahua Apaza ${ }^{1}$

\begin{abstract}
RESUMEN
El presente artículo pretende demostrar la factibilidad de la aplicación de la metodología TPM-Lean Manufacturing en las pymes fabricantes de piezas metalmecánicas, ya que existe un crecimiento en la demanda, visible en el sector, que no puede ser aprovechado por sus bajos valores de eficiencia general de los equipos (OEE, por sus siglas en inglés). Se desea contribuir al mejor conocimiento de la técnica de aplicación con el fin de reducir desperdicios en las industrias de procesos para que, a través de un análisis de sus datos de producción, se identifiquen los problemas que les impiden ser eficientes y mediante la selección, desarrollo e implementación de las técnicas de la metodología Lean Manufacturing se consiga orientar a las pymes a realizar acciones de mejora en sus sistemas productivos a un bajo costo.
\end{abstract}

Palabras clave: Manufactura esbelta; industrias de procesos; mantenimiento productivo total; desperdicios; OEE.

\section{INTRODUCCIÓN}

Con el advenimiento de la globalización, las organizaciones manufactureras tuvieron que adaptar sus estrategias de manufactura a los cambios de la economía global. La competencia creciente y el rápido posicionamiento estratégico entre las compañías de la misma industria encausaron a las organizaciones a mejorar, con recursos limitados, sus parámetros de calidad y productividad. Así, muchas organizaciones han comprendido que su supervivencia en el negocio depende fundamentalmente de producir bienes y servicios de alta calidad.

En la presente investigación se demuestra la hipótesis de que el uso y desarrollo de la metodología TPM-Lean Manufacturing mejora la eficiencia general de los equipos (OEE) de la producción, teniendo en cuenta el análisis de fallas y confiabilidad de los equipos además del pronóstico de la demanda de repuestos en una empresa metalmecánica.

Por lo tanto, el aporte del presente estudio es mostrar cómo la utilización de la metodología TPM-Lean Manufacturing, incrementa la OEE, relacionando el cumplimiento de los mantenimientos preventivos con el pronóstico de la producción futura y enfocándose en mejorar el factor calidad al reducir los defectos en los productos fabricados. El objetivo de esta metodología se resume en lograr empresas eficientes de calidad mundial con una inversión menor.

Este artículo está elaborado para ser utilizado por las diferentes compañías del sector manufacturero, en especial por las pequeñas y medianas empresas (pymes) con la exigencia de recolectar datos como tiempos de proceso por unidad y productos fabricados, tiempos de mantenimiento de los equipos o maquinarias y causas que generan los defectos en los productos fabricados.

1 Ingeniera industrial por la Universidad Peruana de Ciencias Aplicadas (Lima, Perú). Actualmente es jefa de producción en la empresa metalmecánica Frecep SAC (Lima, Perú). ORCID: https://orcid.org/0000-0002-1189-8376

Autor de correspondencia: suministrosproyectoss@gmail.com 
El estudio es cuantitativo para alcanzar una profundidad de análisis descriptivo y preexperimental. Descriptivo porque se centra en describir los datos, procesos, o cualquier otro elemento que se someta a un análisis; y preexperimental porque se enfoca en demostrar que la variable independiente modifica la variable dependiente.

\section{Investigación bibliográfica}

\section{Lean Manufacturing}

Lean Manufacturing surgió como una solución para reducir el desperdicio en los procesos de producción implementando los conceptos originados en el sistema de producción de Toyota (TPS, por sus siglas en inglés), que fueron desarrollados por Eiji Toyoda, Taiichi Ohno y Shigeo Shingo a comienzos de los años cuarenta. La aplicabilidad a industrias discretas, es decir, industrias de ensamblaje, ha sido directa. Sin embargo, la aplicabilidad en las industrias de procesos, es decir, las industrias continuas, aún están en desarrollo. Recientemente, estudiosos como Abdulmalek y Rajgopa (2007); King (2019), Mahapatra y Mohanty (2007); Marodin et al. (2018); y Pool et al. (2011), han estado analizando e implementando algunos de los conceptos Lean en las industrias de procesos y han alcanzado resultados notables. El objetivo de Lean Manufacturing es reducir los despilfarros en cada etapa del proceso de producción.

Para Hu et al. (2015), la metodología Lean Manufacturing se aplica cada vez más en compañías manufactureras, independientemente de su tamaño, a fin de volverlas más competitivas y sostenibles y de que logren posicionamiento en el mercado moderno; asimismo la aplicación de esta metodología resultó en una gran agilidad en los procesos de producción. Por lo tanto, la aplicabilidad de Lean en pymes se reconoce como crucial para el desarrollo de economías en todo el mundo.

Powell et al. (2009) reconocen que, aunque muchas de las herramientas y prácticas Lean se han aplicado con éxito en todos los tipos de procesos industriales, hay una notable falta de la aplicación de prácticas de control de producción Lean en industrias del tipo proceso.

Abdulmalek y Rajgopal (2007) presentan un enfoque basado en casos para demostrar cómo las prácticas de manufactura esbelta (Lean Manufacturing), cuando se usan apropiadamente, ayudan a eliminar desperdicios en las industrias de procesos, mantienen un mejor control de inventarios, mejoran la calidad del producto y logran que se obtenga un mejor control financiero y operacional general. No todas las herramientas Lean son aplicables en los mismos entornos de fabricación; por lo tanto, se debe identificar qué herramientas Lean son aplicables en cada entorno de fabricación dentro de los sectores de proceso.

Existen metodologías magras que son aplicables a todos los tipos de entornos de fabricación en el sector de procesos, independientemente de sus características específicas. Estas herramientas ofrecen la posibilidad de obtener ganancias significativas con inversiones relativamente bajas, ya que requieren una aplicación simple (Abdulmalek y Rajgopal, 2007; Powell et al., 2009).

La flexibilidad de fabricación es muy importante para la agilidad y se puede mejorar mediante una implementación Lean adecuada. Significa que las industrias construyen lo que el cliente pide lo antes posible después del pedido (Saleeshya et al., 2012).

\section{TPM y OEE}

Según Jasiulewicz-Kaczmarek (2016), el mantenimiento productivo total (TPM, por sus siglas en inglés) es un conjunto de iniciativas estratégicas centradas en mantener y mejorar los sistemas de producción y calidad a través de las máquinas, equipos, procesos y empleados que agregan valor a una organización.

EI TPM tiene ocho pilares destinados principalmente a mejorar de manera proactiva la confiabilidad de las máquinas. Las personas están en el centro de este sistema y deben recibir capacitación continua para identificar y eliminar el desperdicio (Zarreh et al., 2018).

Las publicaciones sobre TPM coinciden en que esta metodología posee una filosofía de trabajo participativa, cuyo objetivo es maximizar la efectividad de las maquinarias y equipos eliminando errores, defectos y otros fenómenos negativos.

Según Moreira et al. (2018), OEE es un indicador de rendimiento que permite medir la eficiencia global del equipo. Con él, es posible saber cuál es la eficiencia productiva de una empresa, departamento o máquina.

Nakajima (1988) señala seis tipos de pérdidas y las conglomera en tres grupos, asociándolas individualmente a un indicador; así, menciona que existen: (a) pérdidas por la falta de disponibilidad de equipos, (b) pérdidas por bajo funcionamiento o rendimiento de los equipos y (c) pérdidas por fabricar productos de baja calidad. Asocia estos grupos 
a sus indicadores disponibilidad $(A)$, desempeño $(P)$ y calidad $(Q)$ respectivamente.

Al efectuar la operación de multiplicación de los tres indicadores mencionados, se obtiene el indicador de eficiencia global del equipo (OEE). La Figura 1 ilustra el procedimiento para realizar los cálculos de la OEE y muestra su relación con los seis tipos de pérdidas mencionadas. Ante la aparición de nuevos esquemas productivos con sistemas cada vez más complejos, el cálculo de la OEE debe también ser apoyado por tecnologías nuevas con la finalidad de garantizar la calidad de la información, "de igual manera éste debe estructurarse bajo una buena estrategia de manufactura como lo es la filosofía "Lean" y debe de ser complementado por la metodología de mejora continua" (García et al., 2011).

\section{Análisis de fallas}

La distribución de Weibull es una de las distribuciones más utilizadas en ingeniería de confiabilidad y mantenimiento. Dependiendo del valor del parámetro de forma, $\beta$ (beta), la tasa de fallas puede decrecer, ser constante o crecer. Se utiliza para modelar el comportamiento de falla de varios sistemas de vida real. (Chaurasiya et al., 2017; Bistouni y Jahanshahi, 2015).

\section{MTBF y MTTR}

Según Saetta, y Caldarelli, (2018), el tiempo medio entre fallas (MTBF) es un KPI que se refiere al tiempo de producción promedio transcurrido entre una falla y la próxima vez que ocurre.

$$
\text { MTBF }=\frac{\text { Tiempo disponible de producción }}{\text { Paradas de máquinas }+ \text { Otras paradas }}
$$

Asimismo, indican que el tiempo medio de reparación (MTTR) es el tiempo que lleva ejecutar una reparación después de la ocurrencia de la falla. Es decir, es el tiempo empleado durante la intervención en un proceso dado.
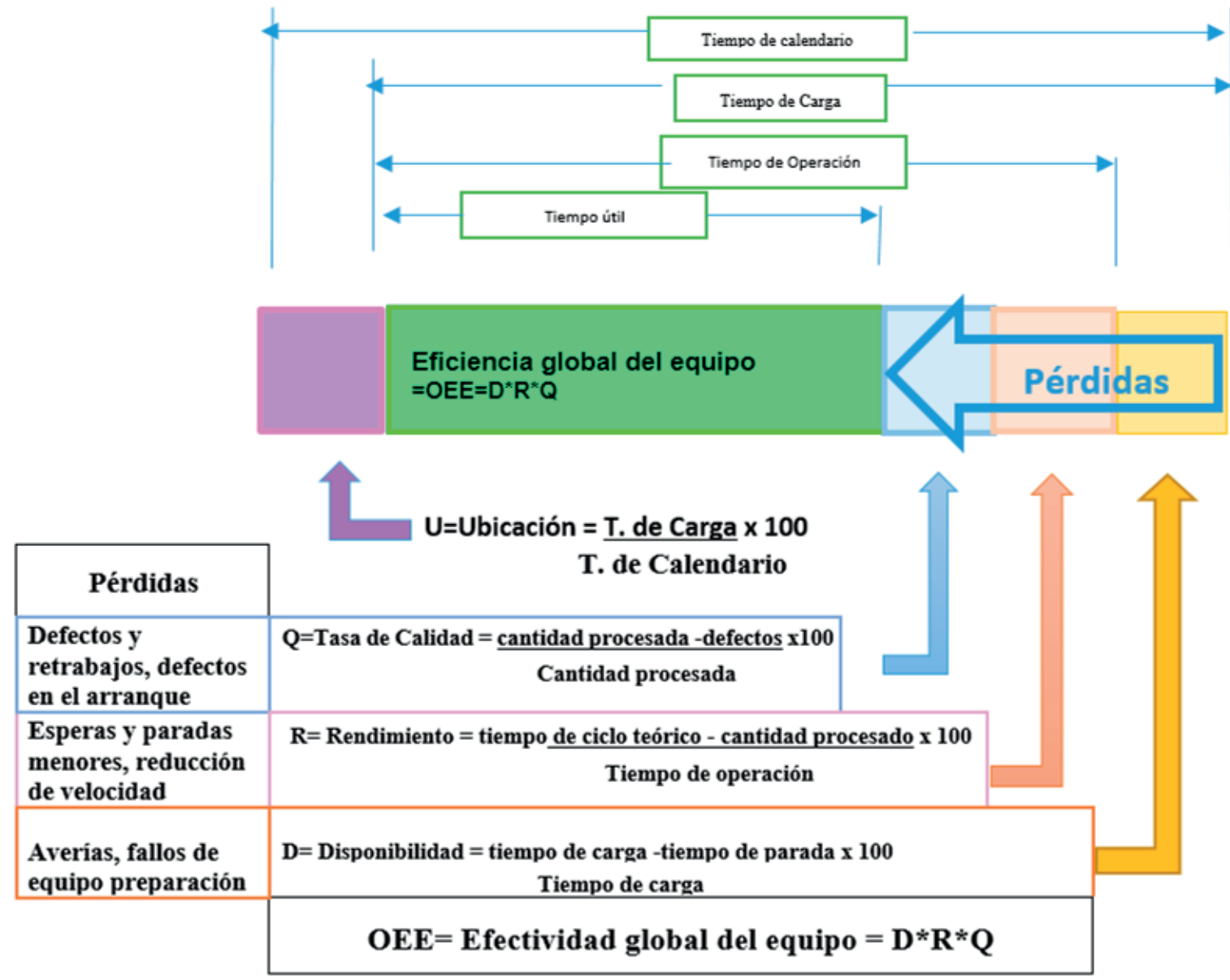

Figura 1. Eficiencia Global del Equipo OEE y su vínculo con las seis grandes pérdidas en la producción.

Fuente: García et al. (2011). 
MTTR $=\frac{\text { Tiempo de paradas de máquinas }+ \text { Tiempo de otras paradas }}{\text { Paradas de máquinas }+ \text { Otras paradas }}$

\section{METODOLOGÍA}

Hernández et al. (2010) afirman que "El investigador puede incluir en su estudio dos o más variables independientes. Cuando en realidad existe una relación causal entre una variable independiente y una dependiente, al variar intencionalmente la primera, la segunda también variará" (p. 54); por ejemplo, si existe una relación causal entre la metodología TPM y el OEE, la implementación de la TPM afectará la OEE, por lo que se puede decir que esta investigación es preexperimental.

Según Tamayo (2003), la investigación experimental puede presentar diversos tipos de diseños experimentales, siendo el más conocido el diseño preexperimental; por lo tanto, el diseño de esta investigación es preexperimental, debido a que se cuenta con un grupo de control pretest y postest.

Hernández et al. (2010), señalan que la investigación cuantitativa "usa la recolección de datos para probar hipótesis, con base en la medición numérica y el análisis estadístico, para establecer patrones de comportamiento y probar teorías" (p. 4).

Tamayo (2003) afirma que "la investigación cuantitativa consiste en el contraste de teorías ya existentes a partir de una serie de hipótesis surgidas de la misma". Este autor también destaca "la necesidad de trabajar con una muestra representativa de una población o fenómeno objeto de estudio".

Cauas (2006) plantea que se "utiliza la recolección y el análisis de datos para contestar preguntas de investigación y probar hipótesis establecidas previamente, confía en la medición numérica, el conteo y frecuentemente en el uso de estadísticas para establecer con exactitud, patrones de comportamiento en una población" (p. 39).

De acuerdo con Hernández et al. (2010), la investigación descriptiva busca especificar propiedades, características y rasgos importantes de cualquier fenómeno que se analice.

Según Tamayo (2003), "el tipo de investigación descriptiva comprende el registro, análisis e interpretación de la naturaleza actual, y la composición o procesos de los fenómenos". El autor aconseja trabajar sobre las realidades de hecho y su característica fundamental de presentar una interpretación correcta.
Para verificar que la metodología Lean sea aplicable en la solución de problemas en una empresa de procesos pyme, hemos seleccionado para el estudio a la empresa Frecep SAC, fabricante de repuestos para máquinas y equipos mineros, la cual opera en la ciudad de Lima.

La empresa metalmecánica presenta una gran cantidad de entregas de productos terminados con demoras excesivas a sus clientes, lo que crea insatisfacción por parte de estos. Como consecuencia, crece la pérdida de clientes, se incrementan los costos de producción, se reducen las utilidades y se presenta la amenaza de ser excluida del mercado.

La presente investigación es un estudio descriptivo y preexperimental de enfoque cuantitativo, puesto que se trabaja con la base de datos proporcionada por la empresa, con la que se realiza un análisis y medición de las mejoras implementadas.

Como Lean Manufacturing es una filosofía de trabajo que busca mejorar y optimizar cualquier sistema de producción enfocándose en la identificación y eliminación de desperdicios, que son las actividades que consumen más recursos de los necesarios, aplicaremos la metodología sugerida por los diferentes autores consultados en el caso de estudio que nos ocupa.

Como fuente de datos principal, se analizó la información sobre la producción del año 2018 proporcionada por la empresa metalmecánica para que, una vez analizada, nos permitiera seleccionar las herramientas operativas que sustenten la aplicabilidad de la metodología Lean para el caso. Así detectamos que la empresa en estudio presenta el problema de baja eficiencia general de equipos (OEE) en la línea de producción de repuestos, lo cual genera costos innecesarios debido principalmente al incumplimiento del programa de mantenimiento preventivo, lo que a su vez genera numerosas paradas de sus máquinas e incrementa los trabajos de reparaciones por mantenimiento correctivo; en consecuencia, se obtienen productos finales de baja calidad y las entregas de repuestos fabricados a destiempo.

\section{ANÁLISIS}

\section{Unidad de análisis}

La unidad de análisis del presente trabajo de investigación es una pieza fabricada en la empresa metalmecánica en estudio. 


\section{Población de estudio}

La población elegida para este estudio fue el total de la producción de repuestos del año 2018, es decir, las 789 piezas fabricadas, según se indica en la Tabla 1.

\section{Tamaño de muestra}

Para el cálculo del tamaño de la muestra se ha considerado una población $\mathrm{N}$ de 789 piezas (producción total del año 2018). El valor de $Z$ se refiere al nivel de confianza, el cual ha sido seleccionado al 95\%; en consecuencia, el error (E) será de 0.05 . En la Tabla 2 se muestra el cálculo del tamaño de muestra.

\section{Selección de muestra}

La muestra se ha seleccionado de la base de datos históricos con respecto y únicamente a aquellas piezas fabricadas con defectos. La muestra representa el $57.71 \%$ de la población, según se indica a continuación en la Tabla 3.

Tabla 1. Producción del año 2018.

\begin{tabular}{|lc|}
\hline Producción & Frecuencia \\
\hline Bocinas & 38 \\
Eje & 57 \\
Eje piñón & 39 \\
Ejes de motor & 76 \\
Engranajes de motor & 96 \\
Engranajes helicoidales & 173 \\
Engranajes rectos & 135 \\
Piñones rectos & 40 \\
Sistemas de transmisión & 115 \\
Soportes & 20 \\
\hline Total & 789 \\
\hline
\end{tabular}

Fuente: Elaboración propia.

Tabla 2. Cálculo de muestra.

\begin{tabular}{clc}
\hline $\mathrm{n}$ & Muestra & ¿X? \\
$\mathrm{Z}$ & Constante de nivel de confianza & 1.95 \\
& Nivel de confianza & $95 \%$ \\
$\mathrm{p}$ & Individuos que poseen la característica de estudio & 0.5 \\
$\mathrm{q}$ & $1-\mathrm{p}$ & 0.5 \\
$\mathrm{~N}$ & Población & 789 \\
$\mathrm{e}$ & Error de muestra & 0.05 \\
$\mathrm{n}=$ & & 247 \\
\hline
\end{tabular}

Fuente: Elaboración propia.

Tabla 3. Tipos de Fallas.

\begin{tabular}{|lccc|}
\hline \multicolumn{1}{|c}{ Causas de las piezas defectuosas } & Subcausas & Frecuencia & Total \\
Mala medición y corte & Falla de máquina & 71 & 122 \\
Deficiente torneado & Falla de máquina & 75 & 138 \\
Deficiente fresado & Falla de máquina & 45 & 83 \\
Deficiente rectificado & Falla de máquina & 56 & 85 \\
Total & & 247 & 428 \\
Equivalente & & $57.71 \%$ & \\
\hline
\end{tabular}

Fuente: Elaboración propia. 


\section{Técnicas de recolección de datos}

Se utilizó la base de datos de la empresa, que fue proporcionada por el área de planeamiento y control de la producción. Los reportes de producción, de fallas de los equipos y los registros de mantenimientos preventivos y correctivos fueron usados para describir los procesos y analizar las variables provistas, que correspondían a la línea de producción de repuestos para equipos del sector minero de dicha empresa metalmecánica. En la Figura 2 se muestra el esquema que se utilizó para el procesamiento y análisis de datos.

Al analizar la información, se ha detectado que la operación de la empresa en estudio se caracteriza por los siguientes resultados:

- Del total de 789 unidades producidas en el año 2018, se entregaron a destiempo 340 , que representan el $43.09 \%$ de la producción anual.
- El incumplimiento del programa de mantenimiento preventivo para el año 2018 fue del $93.75 \%$, pues de 16 trabajos de mantenimientos preventivos programados, 4 para cada una de las máquinas (cortadora, torno, fresadora y rectificadora), solo se cumplió 1.

- El exceso de horas de mantenimiento correctivo para el año 2018 es de $96.76 \%$, es decir, 239 procesos de los 247 procesos seleccionados como muestra excedieron las horas programadas para cada mantenimiento correctivo.

- Se generaron reprocesos y mermas como productos no conformes: para la producción de 789 unidades se generaron 414 reprocesos durante la fabricación, y se obtuvieron 393 productos como merma, por lo que se asume que se abrieron 1596 procesos nuevos para producir 789 unidades, los reprocesos

PROCESAMIENTO Y ANÁLISIS DE DATOS

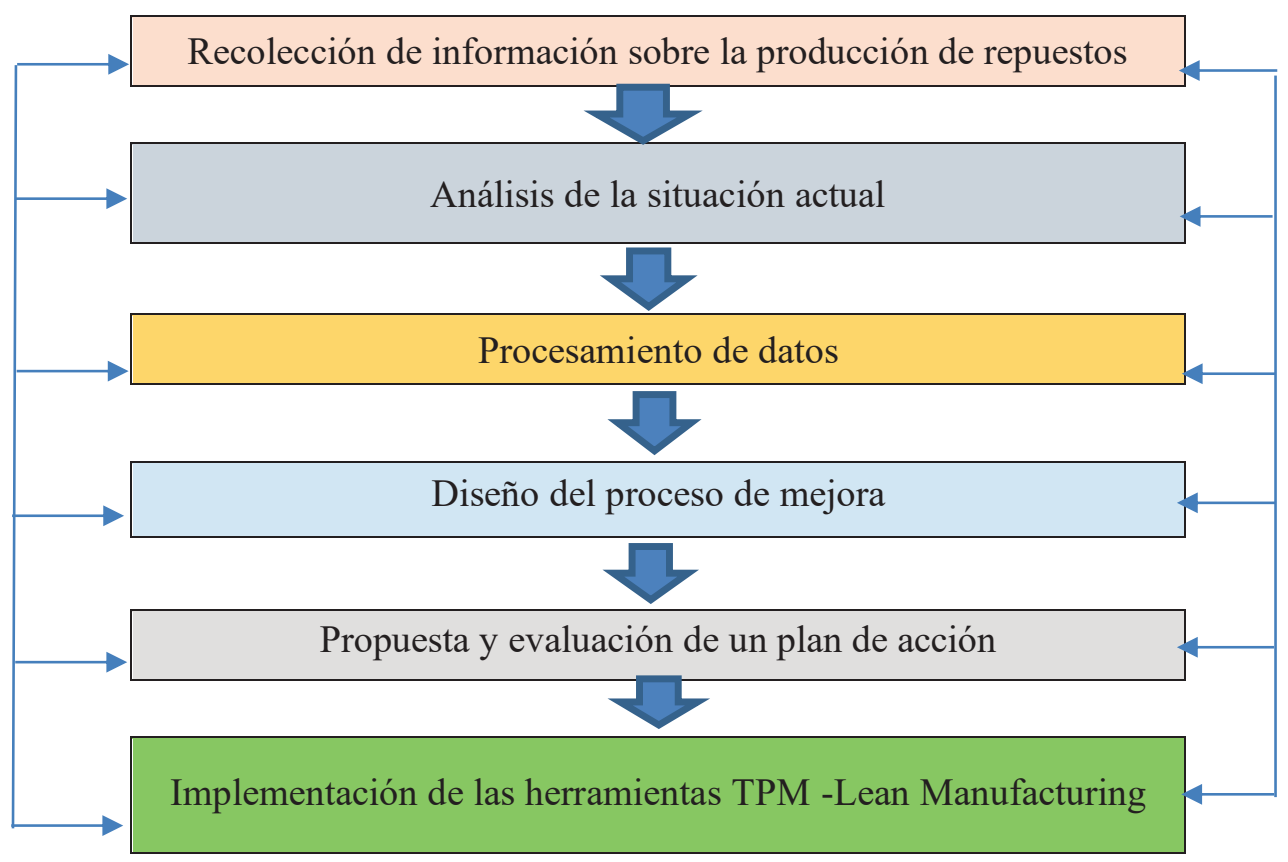

Figura 2. Esquema de procesamiento y análisis de datos.

Fuente: Elaboración propia. 
equivalen al $25.94 \%$, y los procesos que generaron merma equivalen a $24.62 \%$ y los productos entregados equivalen a $49.44 \%$ de los 1596 procesos.

- Los problemas ocurrieron por los trabajos realizados con deficiencia en las áreas de medición y corte, torneado, fresado y rectificado. En ellos tiene gran incidencia la ocurrencia de fallas en las máquinas $(58.2 \%$ en medición y corte; $54.35 \%$ en torneado; $54.22 \%$ en fresado y $65.8 \%$ en rectificado).

Se infiere que la falla de los equipos se origina por la falta de ejecución de trabajos de mantenimiento preventivo en ellos, lo cual ha generado pérdidas de tiempo, recursos, materiales y dinero; asimismo, la utilización de recursos humanos adicionales para cubrir inspecciones en reprocesos significa una gran pérdida en la productividad.

Identificado el problema, seleccionamos al mantenimiento productivo total y a la detección, prevención y eliminación de desperdicios como técnicas de la metodología Lean Manufacturing que se utilizarán para mejorar el rendimiento de la empresa en estudio.

\section{Planteamiento y prueba de hipótesis}

A fin de lograr el procesamiento de los datos para el estudio actual, usamos el programa informático Excel de Microsoft, específicamente sus herramientas estadísticas y aquellas creadas para construir tablas y gráficos personalizados. Para la interpretación de los resultados estadísticos, utilizamos los libros de Chue et al. (2009) y Córdova (2003).
Se hicieron las pruebas de hipótesis del análisis de fallas, confiabilidad y demanda, así como la de la hipótesis general, la cual, para ser validada, requiere de la validación previa de las tres anteriores. Mostraremos los resultados de la prueba de hipótesis general, pues las cuatro mencionadas tiene el mismo esquema.

\section{Prueba de hipótesis general}

Esta hipótesis se debe validar por medio de las tres hipótesis específicas, ya que estas se requieren para aceptar o rechazar la hipótesis general por medio de los valores de OEE.

Hipótesis nula $-\mathrm{H}_{0}$ : La utilización de la metodología TPM-Lean Manufacturing no mejora la OEE en la producción de repuestos para equipos del sector minero en la empresa Frecep SAC.

Hipótesis alterna $-\mathrm{H}_{1}$ : La utilización de la metodología TPM-Lean Manufacturing sí mejora la OEE en la producción de repuestos para equipos del sector minero en la empresa Frecep SAC.

Para validar las hipótesis planteadas, utilizaremos una prueba $t$ de student para muestras emparejadas, seleccionando una confiabilidad del 95\%. Una vez obtenido el resultado, la hipótesis nula se rechazará si $\mathrm{t}<-2.776$ y si $\mathrm{t}>2.776$. Utilizaremos los datos tomados antes y después de la implementación de TPM en cuanto a los valores de OEE actual y mejorada, como se muestra en la Tabla 4.

Donde, $\mathrm{t}$ stat $=-20.446$, es decir, es menor que -2.776; entonces, la hipótesis nula es rechazada y se acepta la hipótesis alterna. Dado que existe un

Tabla 4. Análisis t de Student - Hipótesis general.

\begin{tabular}{|lcc|}
\hline \multicolumn{1}{|c|}{ t-Test: Paired Two Sample for Means } & Actual & Mejora \\
\hline Media & 133.0000000 & 65.3352210 \\
Varianza & 423.0909091 & 4.9223734 \\
Observaciones & 12 & 12 \\
Correlación de Pearson & 0.5808295 & \\
Diferencia Media Hipotética & 0 & \\
Df & 11 & \\
t Stat & 12.1042398 & \\
$\mathrm{P}(\mathrm{T}<=\mathrm{t})$ una-cola & 0.0000001 & \\
t Crítico una-cola & 1.7958848 & \\
$\mathrm{P}(\mathrm{T}<=\mathrm{t})$ dos-colas & 0.0000001 & \\
$\mathrm{t}$ Crítico dos-colas & 2.2009852 & \\
\hline
\end{tabular}

Fuente: Elaboración propia. 
nivel de confianza del 95\%, procedemos a aceptar la hipótesis general alterna que asevera: "La utilización de la metodología TPM-Lean Manufacturing sí mejora la OEE en la producción de repuestos para equipos del sector minero en la empresa Frecep SAC".

\section{Aplicación de la técnica TPM}

Para la implementación de TPM ejecutamos el pilar de mantenimiento planificado y el pilar de mejora enfocada, la cual se refiere a analizar las fallas y mejorar el proceso de gestión del mantenimiento. Al analizar el cronograma de los mantenimientos preventivos, observamos que en el año 2018 no se realizaron mantenimientos planificados (MP) a los equipos cortadora, torno y fresadora. Por el incumplimiento de MP, que fue del $93.75 \%$, se ocasionó un quiebre en la operación, lo que originó una serie de mantenimientos correctivos (MC). Al cumplirse sólo 8.47 horas de MP, se originaron 1917.33 horas de MC y 1177.64 horas en exceso de MC, es decir que por cada MP realizado se ejecutaron $226 \mathrm{MC}$ aproximadamente.

Se generó una hoja de Excel para determinar el eje $x$ y eje $y$, a fin de utilizar la herramienta de regresión y así calcular los parámetros de fallas de Weibull $\beta$ y $\alpha$, utilizando las ecuaciones de Weibull.

Así mismo, se calcularon los indicadores MTBF y MTTR: El valor de MTBF fue de 50.86 horas, lo que significa que cada 50.86 horas ocurre una falla en cualquiera de los 4 equipos, y el tiempo medio de reparación por cada $\mathrm{MC}$ es de 7.76 horas como promedio. Con estos datos podemos calcular la confiabilidad de los equipos como se muestra en la Tabla 5.

El cálculo de la confiabilidad de la línea es bajo, ya que a las 6 horas es de 0.7139 , a las 10 horas solo es de 0.2668 y a las 19 horas después de una falla es de 0.

Al aplicar el pilar de mejora enfocada, generamos un nuevo cronograma de mantenimiento preventivo en el que consideramos que, debido al desgaste de los equipos, se calcula que cada 13 días, (MTBF/8 horas por día) se debe realizar un mantenimiento preventivo. Se ha considerado el trabajo de 365 días, por lo que hay una frecuencia de $28 \mathrm{MP}$ en un año.

Se ha calculado que la probabilidad de ejecución de un MP es de 0.25 , por lo que se asume que de una frecuencia de $28 \mathrm{MP}$ para cada uno de los equipos, se deben ejecutar 7 , y en el caso del mantenimiento autónomo (MA), se deben ejecutar 13. Es decir, que al programarse un total de 320 mantenimientos, entre MP y MA, se deben ejecutar 80 . La planificación de los trabajos de MP es complementada con la creación de los formatos y bitácoras a utilizar para registrar las verificaciones en la ejecución de los mismos.

En base a la demanda actual (año 2018) se realizó un pronóstico de la demanda futura con las condiciones mejoradas (año 2019). Así, se ha calculado que la demanda diaria de productos es de un promedio de 3.52 unidades por día para el año 2018 y un promedio de 3.29 unidades por día para el año 2019. Considerando que la producción por

Tabla 5. Cálculo de confiabilidad - línea de producción (4 equipos).

\begin{tabular}{|l|c|c|c|c|}
\hline \multicolumn{5}{|c|}{ CÁLCULO DE CONFIABILIDAD - LÍNEA DE PRODUCCIÓN (4 EQUIPOS) } \\
\hline Beta (parámetro pendiente) & & Horas & Probabilidad falla & Confiabilidad \\
\hline Eta (Característica vida) = & 2.91 & 0 & 0.0000 & 1.0000 \\
\hline MTBF (Horas) = & 8.72 & 1 & 0.0031 & 0.9969 \\
\hline MTTR (horas) = & 50.86 & 2 & 0.0232 & 0.9768 \\
\hline & 7.76 & 4 & 0.0735 & 0.9265 \\
\hline & & 5 & 0.1615 & 0.8385 \\
\hline & & 6 & 0.2861 & 0.7139 \\
\hline & & 7 & 0.4359 & 0.5641 \\
\hline & & 10 & 0.5919 & 0.4081 \\
\hline
\end{tabular}

Fuente: Elaboración propia. 
producto es de 1.52336 días, el tiempo de producción para los diferentes productos para el año 2019 es muy similar al utilizar los cuatro equipos para la fabricación.

Se obtuvo una mejora en la disminución del tiempo de entrega de productos terminados a tiempo, que fue un total de 719 unidades. Es decir, que, de la producción anual de 742 unidades, se entregó con demora 23 unidades, debido a reprocesos (22) y procesos que generaron merma (20). Así, se redujeron los operarios de un promedio de 8 a 6 , así como las horas teóricas de 17908 a 12420 horas (por la diferencia en el número de operarios y disminución de horas de ejecución de mantenimientos); las horas programadas se redujeron de 14700 a 10200 horas por año; y las horas de clases y reuniones de 220.50 a 153 horas. Por su parte, se incrementó la cantidad de horas de MP de 8.47 a 247.90 horas, lo que redujo el MC de 1917.33 a 65.51 horas, con lo cual se eliminaron las horas extras de MC. La demora en producción se redujo de 2156.49 a 112.37 horas y la producción se redujo de 9602.80 a 9028.33 horas. Los tiempos muertos se redujeron de 794.40 a 592.89 horas al agregar 40 horas de MA.
Al cumplir con los MP se reducirán los MC, ya que, por la falta de los primeros, ocurren las fallas mecánicas de los equipos y la necesidad de los segundos; por esto, al pronosticar la frecuencia y horas de MP y MC, se ha calculado el siguiente OEE:

En consecuencia, al mejorar el cumplimiento de los MP, y MA en un $30.84 \%$, se ha mejorado el factor calidad de $49.44 \%$ a $94.64 \%$, el factor rendimiento de $76.68 \%$ a $93.34 \%$, y, por ende, se incrementó también el factor disponibilidad de $86.70 \%$ a $96.88 \%$. Se logró incrementar la OEE de $32.86 \%$ a $85.58 \%$, excediendo el índice mundial de $85 \%$. Se llegó a la conclusión de que el incumplimiento de los MP genera los MC y, por ende, resulta en la demora de entrega del proyecto. El cambio de los índices de MTBF y MTTR también son importantes, tal como se muestra en la Tabla 6.

Al calcular el MTBF anual, se observa que se ha incrementado de 50.86 horas a 237.65 horas y se ha reducido el MTTR de 7.76 horas a 0.27 horas.

Es posible cuantificar los ahorros obtenidos por la implementación del TPM, los cuales ascienden a S/ 590353.55 como consecuencia de reducir el número de operarios y las horas y demora por MC. Por su parte, la inversión es de S/119 317.15 por el

Tabla 6: Cálculo OEE Actual - Mejora.

\begin{tabular}{|c|c|c|c|c|c|c|c|c|}
\hline \multicolumn{5}{|c|}{ OEE actual } & \multicolumn{4}{c|}{ OEE mejora } \\
\hline Disponibilidad & Rendimiento & Calidad & OEE & Disponibilidad & Rendimiento & Calidad & OEE \\
\hline \multicolumn{7}{|c|}{ Estándar World Class } & \multicolumn{3}{c|}{ Estándar World Class } \\
\hline $90 \%$ & $95 \%$ & $99 \%$ & $\mathbf{8 5 \%}$ & $90 \%$ & $95 \%$ & $99 \%$ & $85 \%$ \\
\hline $66.16 \%$ & $76.41 \%$ & $48.78 \%$ & $24.66 \%$ & $87.60 \%$ & $90.30 \%$ & $94.10 \%$ & $74.43 \%$ \\
\hline $90.43 \%$ & $72.86 \%$ & $47.41 \%$ & $31.24 \%$ & $93.72 \%$ & $97.43 \%$ & $93.84 \%$ & $85.69 \%$ \\
\hline $89.51 \%$ & $79.16 \%$ & $52.50 \%$ & $37.20 \%$ & $85.26 \%$ & $88.29 \%$ & $95.40 \%$ & $71.82 \%$ \\
\hline $83.20 \%$ & $72.65 \%$ & $45.07 \%$ & $27.24 \%$ & $88.50 \%$ & $92.23 \%$ & $94.22 \%$ & $76.90 \%$ \\
\hline $88.32 \%$ & $82.45 \%$ & $54.62 \%$ & $39.77 \%$ & $85.34 \%$ & $88.76 \%$ & $95.43 \%$ & $72.29 \%$ \\
\hline $86.79 \%$ & $81.44 \%$ & $50.40 \%$ & $35.62 \%$ & $91.84 \%$ & $95.62 \%$ & $95.22 \%$ & $83.62 \%$ \\
\hline $84.25 \%$ & $74.57 \%$ & $48.63 \%$ & $30.55 \%$ & $94.00 \%$ & $98.45 \%$ & $93.85 \%$ & $86.85 \%$ \\
\hline $89.62 \%$ & $74.09 \%$ & $49.34 \%$ & $32.76 \%$ & $87.61 \%$ & $91.40 \%$ & $94.11 \%$ & $75.36 \%$ \\
\hline $83.11 \%$ & $75.67 \%$ & $53.49 \%$ & $33.64 \%$ & $93.99 \%$ & $97.87 \%$ & $96.84 \%$ & $89.08 \%$ \\
\hline $87.82 \%$ & $74.51 \%$ & $48.25 \%$ & $31.57 \%$ & $89.84 \%$ & $94.69 \%$ & $93.61 \%$ & $79.64 \%$ \\
\hline $82.23 \%$ & $78.89 \%$ & $48.74 \%$ & $31.62 \%$ & $91.59 \%$ & $95.53 \%$ & $95.22 \%$ & $83.31 \%$ \\
\hline $87.70 \%$ & $77.45 \%$ & $48.97 \%$ & $33.26 \%$ & $86.28 \%$ & $89.49 \%$ & $94.00 \%$ & $72.58 \%$ \\
\hline $86.70 \%$ & $76.68 \%$ & $49.44 \%$ & $32.86 \%$ & $96.88 \%$ & $93.34 \%$ & $94.64 \%$ & $85.58 \%$ \\
\hline
\end{tabular}

Fuente: Elaboración propia. 
incremento de MP y MA y por la capacitación a los operarios para una debida gestión de los trabajos de mantenimiento. Esto se muestra en la Tabla 7.

\section{RESULTADOS}

Como resultado final, se concluye que al mejorarse el cumplimiento de los MP (mantenimientos preventivos) y MA (mantenimientos autónomos) se han mejorado el factor calidad (de $49.44 \%$ a $94.64 \%$ ), el factor rendimiento (de $76.68 \%$ a $93.34 \%$ ), y, por ende, se incrementó el factor disponibilidad (de $86.70 \%$ a $96.88 \%$ ), con lo que se logró incrementar el OEE de $32.86 \%$ a $85.58 \%$, excediendo el índice mundial de 85. Además, la relación de mantenimientos preventivos y correctivos también mejora de 1:226 a 4:1. En la Tabla 8 se muestran los indicadores.

Los índices de MTBF (tiempo medio entre fallas) y MTTR (tiempo medio para reparar) también son importantes. Al calcular el MTBF anual se nota que su valor se ha incrementado de 50.86 horas a 237.65 horas y se ha reducido el MTTR de 7.76 horas a 0.27 horas, como se muestra en las Tablas 9 y 10.

Por último, se ha cambiado el proceso de gestión de mantenimiento al reducirse la actividad, como se ilustra en la Figura 3, asumiendo que se cumplen los MP, como se ha establecido en esta investigación.

\section{CONCLUSIONES}

Después de haber desarrollado el presente trabajo de investigación para demostrar la factibilidad de la aplicación de la metodología TPM-Lean Manufacturing en las empresas fabricantes de piezas metalmecánicas, se han determinado las siguientes conclusiones:

- El uso y aplicación de la metodología TPM- Lean Manufacturing es conveniente para mostrar el diagnóstico del estado actual y para encontrar y proponer alternativas a fin de lograr la mejora de un proceso de fabricación.

- Es tangible la influencia que el uso de las herramientas de la metodología Lean Manufacturing tiene en el incremento de la eficiencia de la producción de repuestos para equipos del sector minero en la empresa metal mecánica FRESEP SAC.

- Mediante el procesamiento adecuado de una necesaria base de datos, se pueden determinar las causas fundamentales de la baja OEE en la producción de repuestos; estas causas, en el caso de la empresa en estudio, son el incumplimiento de los mantenimientos preventivos y el exceso de horas utilizadas en mantenimientos correctivos, según se obtuvo a través de la utilización de la metodología TPM.

- Mediante la aplicación de la metodología TPM, la OEE en la producción de repuestos para equipos del sector minero en la empresa metal mecánica FRESEP SAC se ha incrementado de $32.86 \%$ a $85.58 \%$.

- Para implementar las mejoras propuestas en la empresa en estudio, se requiere efectuar una

Tabla 7. Ahorros e inversión.

\begin{tabular}{|c|c|c|c|}
\hline \multicolumn{4}{|c|}{ Ahorros } \\
\hline Operarios & 2 (op) & S/ 1750.00 & S/ 42000.00 \\
\hline$M C$ & 1851.82 (hrs.) & & S/ 260644.32 \\
\hline Demora por MC & 2044.12 (hrs.) & & S/ 287709.23 \\
\hline Total & & & S/ 590353.55 \\
\hline \multicolumn{4}{|c|}{ Inversión } \\
\hline MP & 239.43 & & S/ 80677.55 \\
\hline MA & 40 & & S/ 13478.27 \\
\hline Capacitación & 4 & & S/ 21675.00 \\
\hline Costo por hora & 68 & & \\
\hline Horas por op & 75 & & \\
\hline Horas extra & 8.75 & 2.19 & S/ 3486.33 \\
\hline Total & & & S/ 119317.15 \\
\hline
\end{tabular}

Fuente: Elaboración propia. 
Tabla 8. Indicadores.

\begin{tabular}{|llllr|}
\hline \multicolumn{1}{r}{ Indicador } & Actual & \multicolumn{1}{c|}{ Objetivo } & \multicolumn{1}{c|}{ Estándar Mundial } & Mejora \\
\hline OEE & $32.86 \%$ & Incrementar & $85 \%$ & $85.58 \%$ \\
Disponibilidad & $86.70 \%$ & Incrementar & $90 \%$ & $96.88 \%$ \\
Rendimiento & $76.68 \%$ & Incrementar & $95 \%$ & $93.34 \%$ \\
Calidad & $49.44 \%$ & Incrementar & $99 \%$ & $94.64 \%$ \\
& & & Normal 3:1 & 4.1 \\
Relación MP:MC & $1: 226$ & Incrementar MP y & Restrictivo 5:1 & $4: 1$ \\
& & reducir MC & Más restrictivo 6:1 & 4.1 \\
\hline
\end{tabular}

Fuente: Elaboración propia.

Tabla 9. Comparación MTTR y MTBF del proceso actual producción 2018.

\begin{tabular}{|cccccccccc|}
\hline Año & Mes & $\begin{array}{c}\text { Total-Horas } \\
\text { producción }\end{array}$ & Horas fallas & $\begin{array}{c}\text { Frecuencia } \\
\text { de fallas }\end{array}$ & MTBF & MP & MC & MTTR MC \\
\hline & Ene & 1478 & 198 & 22 & 58.15 & 0.00 & 198.15 & 9.01 \\
& Feb & 1182 & 113 & 16 & 66.80 & 0.00 & 113.17 & 7.07 \\
& Mar & 1084 & 114 & 14 & 69.27 & 0.00 & 113.70 & 8.12 \\
& Abr & 1281 & 215 & 30 & 35.51 & 0.00 & 215.07 & 7.17 \\
& May & 1084 & 127 & 16 & 59.81 & 0.00 & 126.56 & 7.91 \\
& Jun & 1084 & 143 & 18 & 52.24 & 0.00 & 143.17 & 7.95 \\
& Jul & 1379 & 217 & 28 & 41.49 & 0.00 & 217.17 & 7.76 \\
& Ago & 1379 & 143 & 18 & 68.66 & 0.00 & 143.15 & 7.95 \\
& Set & 887 & 150 & 18 & 40.93 & 0.00 & 149.69 & 8.32 \\
& Oct & 1281 & 156 & 22 & 51.11 & 0.00 & 155.98 & 7.09 \\
& Nov & 1084 & 184 & 24 & 37.48 & 8.47 & 184.05 & 7.67 \\
& Dic & 1281 & 157 & 21 & 53.48 & 0.00 & 157.48 & 7.50 \\
\hline
\end{tabular}

Fuente: Elaboración propia.

Tabla 10. Comparación MTTR y MTBF del proceso mejorado producción 2019.

\begin{tabular}{|cccccccccc|}
\hline Año & Mes & $\begin{array}{c}\text { Total horas } \\
\text { producción }\end{array}$ & Horas fallas & $\begin{array}{c}\text { Frecuencia } \\
\text { de fallas }\end{array}$ & MTBF & MP & MC & MTTR MC \\
\hline \multirow{8}{*}{ Ene } & 887 & 7 & 4 & 219.93 & 13.40 & 6.77 & 0.31 \\
& Feb & 788 & 4 & 4 & 196.03 & 20.10 & 3.87 & 0.24 \\
& Mar & 887 & 4 & 3 & 294.21 & 20.10 & 3.88 & 0.28 \\
& Abr & 887 & 7 & 4 & 219.79 & 20.10 & 7.35 & 0.24 \\
& May & 887 & 4 & 3 & 294.06 & 23.45 & 4.32 & 0.27 \\
& Jun & 788 & 5 & 3 & 261.04 & 20.10 & 4.89 & 0.27 \\
& Jul & 788 & 7 & 4 & 195.15 & 20.10 & 7.42 & 0.26 \\
& Ago & 887 & 5 & 4 & 220.40 & 23.45 & 4.89 & 0.27 \\
& Set & 788 & 5 & 2 & 391.44 & 20.10 & 5.11 & 0.28 \\
& Oct & 788 & 5 & 4 & 195.67 & 26.80 & 5.33 & 0.24 \\
& Nov & 788 & 6 & 3 & 260.57 & 20.10 & 6.29 & 0.26 \\
& Dic & 887 & 5 & 4 & 220.28 & 20.10 & 5.38 & 0.26 \\
\hline
\end{tabular}

Fuente: Elaboración propia. 


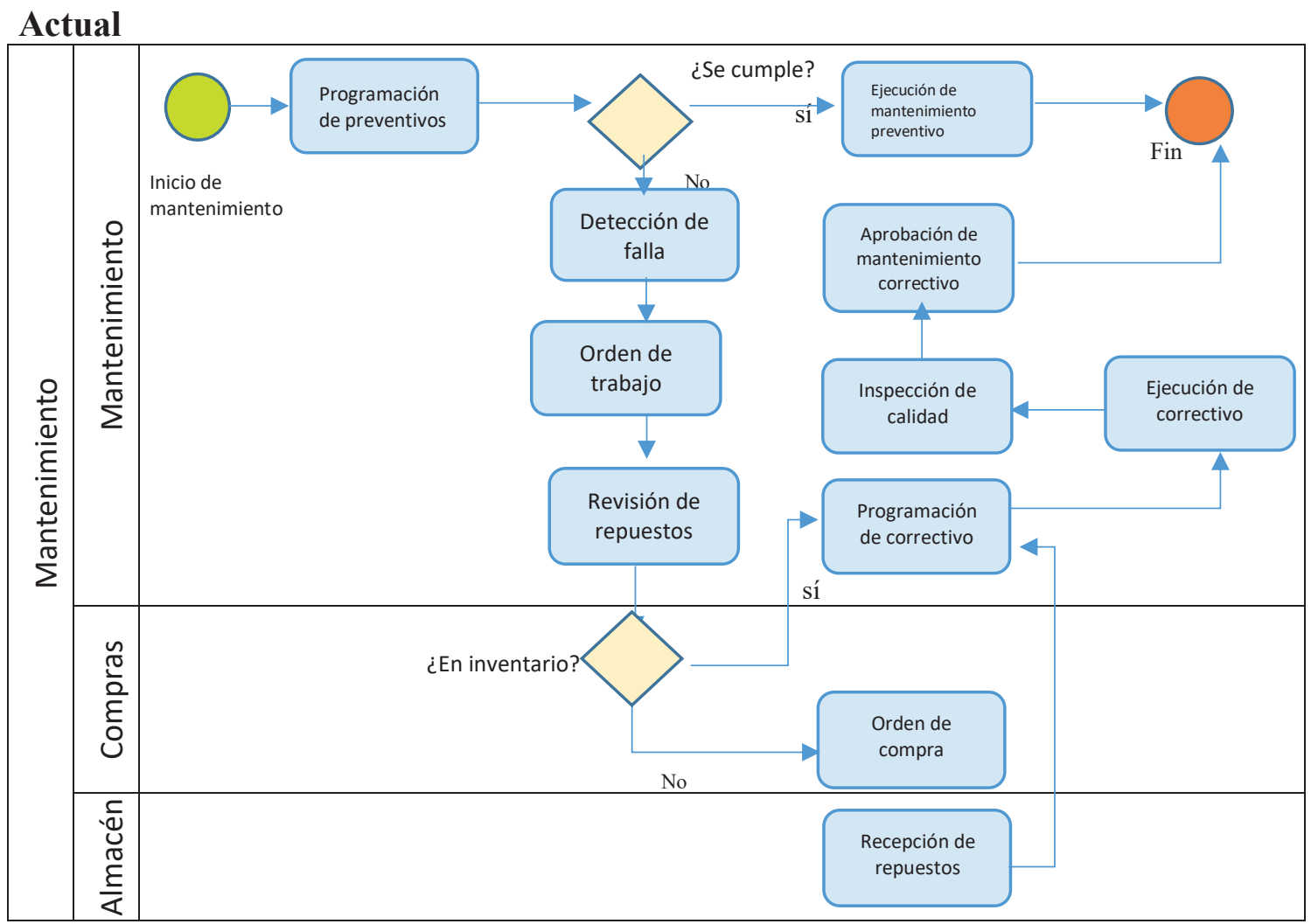

Mejora

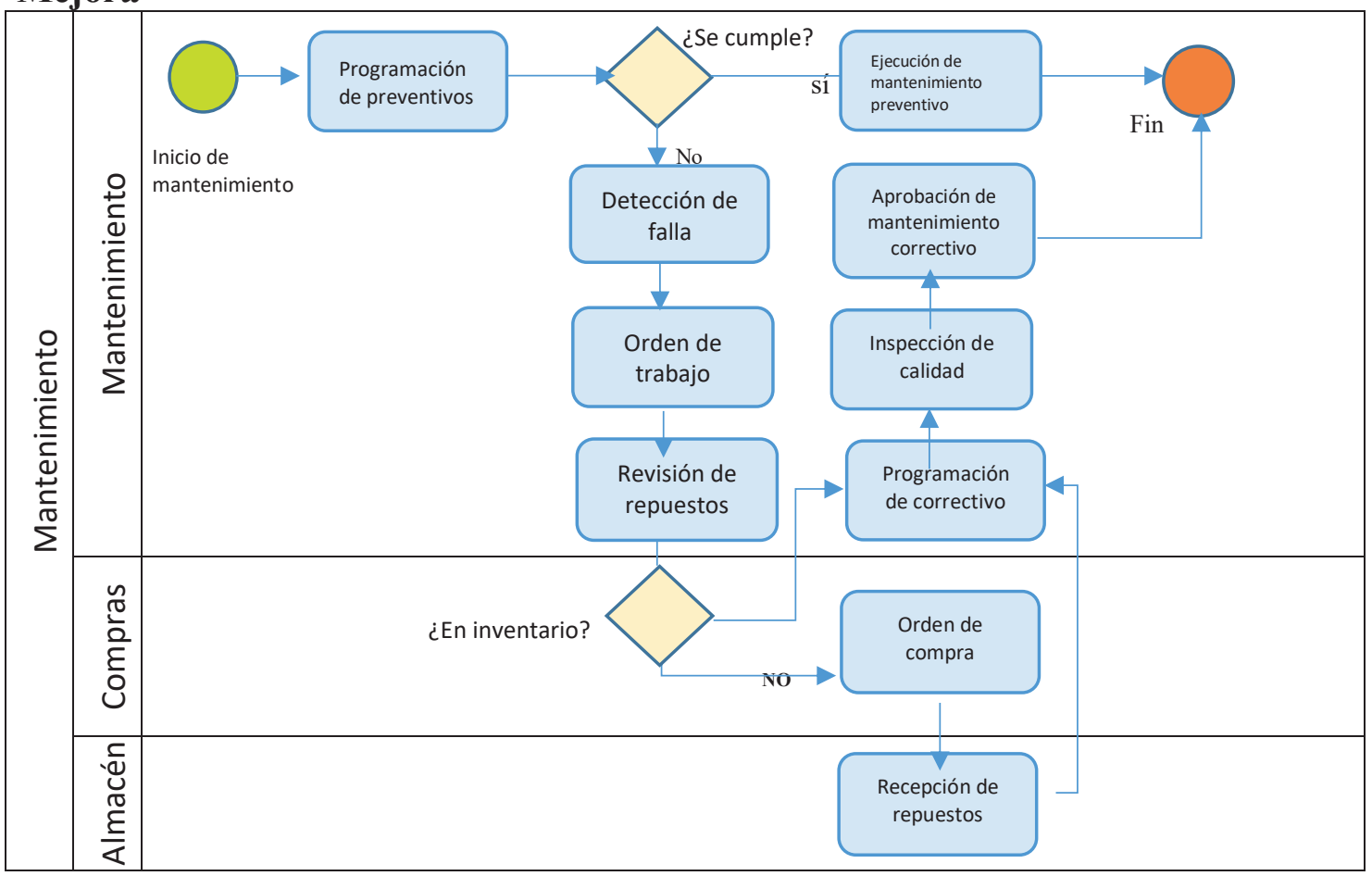

Figura 3. Comparación de Procesos de Gestión de Mantenimientos.

Fuente: Elaboración propia. 
inversión de S/ 119 317.15, lo cual es justificable, pues genera un ahorro de S/ 590353.55 .

- Se han logrado alcanzar los objetivos propuestos y también demostrar las hipótesis planteadas en el presente trabajo de investigación, con lo que se demuestra que su aplicación es factible para las empresas manufactureras del sector PYME a bajos costos.

\section{REFERENCIAS BIBLIOGRÁFICAS}

[1] Abdulmalek, F., y Rajgopal, J. (2007). Analyzing the benefits of lean manufacturing and value stream mapping via simulation: A process sector case study. International Journal of Production Economics, 107(1), 223-236. Recuperado de https://doi.org/10.1016/j.ijpe.2006.09.009

[2] Bistouni, F. y Jahanshai, M. (2015). Evaluating Failure Rate of Fault-Tolerant Multistage Interconnection Networks Using Weibull Life Distribution. Reliability Engineering \& System Safety, 144, 128-146.

[3] Cauas, D. (2006). Elementos para la elaboración y ejecución de un proyecto de investigación. Investigación en Ciencias Sociales. Recuperado de webjam-upload. s3.amazonaws.com/apuntesinvest_ cauas_1539_.pdf

[4] Chaurasiya, P., Ahmed, S., y Warudkar, V. (2017). Wind characteristics observation using Doppler-SODAR for wind energy applications. Resource-Efficient Technologies, 3(4), 495505. Recuperado de https://doi.org/10.1016/j. reffit.2017.07.001

[5] Chue, J., Barreno, E., Castillo, C., Millones, R., y Vásquez, F. (2009). Estadística descriptiva y probabilidades ( $2^{\mathrm{a}}$ ed.). Lima, Perú: Universidad de Lima.

[6] Córdova, M. (2003). Estadística Descriptiva e inferencial ( $5^{\mathrm{a}}$ ed.). Lima, Perú: Pontificia Universidad Católica del Perú.

[7] García, M., Santos, J., Arcelus, M., y Viles, E. (2011). Plug\&Lean-OEE game: Juego de entrenamiento basado en el indicador de efectividad global del equipo enfocado en mejorar la productividad de las operaciones de manufactura. Recuperado de https://www. semanticscholar.org/paper/Plug\%26LeanOEE-game\%3A-Juego-de-entrenamientobasado-Garc\%C3\%ADa-Santos/4c2a517417e 320e6ecee6878e892fad4d625031b
[8] Hernández, R., Fernández, C., y Baptista, P. (2010). Metodología de la investigación (5a ed.). México D.F., México: McGraw-Hill.

[9] Hu, Q., Mason, R., Williams, S.J., y Found, P. (2015). Lean implementation within SMEs: a literature review. Journal of Manufacturing Technology Management, 26(7), 980-1012. Recuperado de https://doi.org/10.1108/JMTM02-2014-0013

[10] Jasiulewicz-Kaczmarek, M. (2016). SWOT analysis for Planned Maintenance strategy-a case study. IFAC-PapersOnLine, 49(12), 674679. Recuperado de https://doi.org/10.1016/j. ifacol.2016.07.788

[11] King, P. L. (2019). Lean for the Process Industries: Dealing with Complexity ( $2^{\mathrm{a}}$ ed.). Nueva York, Estados Unidos: Productivity Press. Recuperado de https://doi. org/10.4324/9780429400155

[12] Mahapatra, S., y Mohanty, S. (2007). Lean Manufacturing in Continuous Process Industry: An Empirical Study. Journal of Scientific \& Industrial Research, 66(1), 1927. Recuperado de http://nopr.niscair.res.in/ handle/123456789/1104

[13] Marodin, G., Frank, A., Tortorella, G., y Netland, T. (2018). Lean Product Development and Lean Manufacturing: Testing Moderation Effects. International Journal of Production Economics, 203, 301-310. Recuperado de https://doi. org/10.1016/j.ijpe.2018.07.009

[14] Moreira, A., Silva, F., Correia, A., Pereira, T., Ferreira, L., y de Almeida, F. (2018). Cost reduction and quality improvements in the printing industry. Procedia Manufacturing, 17(1), 623-630. Recuperado de https://doi. org/10.1016/j.promfg.2018.10.107

[15] Nakajima, S. (1988). Introduction to TPM: Total Productive Maintenance. Cambridge, MA, Estados Unidos: Productivity Press.

[16] Pool, A., Wijngaard, J., y Van Der Zee, D. (2011). Lean planning in the semi-process industry, a case study. International Journal of Production Economics, 131(1), 194-203. Recuperado de https://doi.org/10.1016/j.ijpe.2010.04.040

[17] Powell, D., Alfnes, E., y Semini, M. (2010). The Application of Lean Production Control Methods within a Process-Type Industry: The Case of Hydro Automotive Structures. En B. Vallespir, T. Alix (Eds.), IFIP International Conference on Advances in Production Management Systems. Berlín, Alemania: Springer, Berlin, Heidelberg. 
Recuperado de https://doi.org/10.1007/978-3642-16358-6_31

[18] Saetta, S. y Caldarelli, V. (2018). The machinery performance analysis with smart technologies: a case in the food industry. IFACPapersOnLine, 51(11), 441-446. Recuperado de https://doi.org/10.1016/j.ifacol.2018.08.341

[19] Saleeshya, P. G., Raghuram, P., y Vamsi, N. (2012). Lean manufacturing practices in textile industries - a case study. International Journal of Collaborative Enterprise, 3(1), 1837. Recuperado de https://doi.org/10.1504/ IJCENT.2012.052367
[20] Tamayo M. (2003). El proceso de la investigación científica (4a ed.). México D.F, México: Editorial Limusa, S. A. de C. V. Grupo Noriega Editores.

[21] Zarreh, A., Wan, H., Lee, Y., Saygin, C., y Al Janahi, R. (2018). Cybersecurity Concerns for Total Productive Maintenance in Smart Manufacturing Systems. Procedia Manufacturing, 38(1), 532-539. Recuperado de https://doi.org/10.1016/j.promfg.2020.01.067 


\section{Implementation of the TPM-Lean Manufacturing Methodology to Improve the Overall Equipment Effectiveness (OEE) of Spare Parts Production at a Metalworking Company}

Nohemy Canahua Apaza ${ }^{1}$

\begin{abstract}
This article intends to demonstrate the feasibility of applying the TPM-Lean Manufacturing methodology in SME manufacturers of metal spare parts, as there is a growth in demand, visible in the sector, which cannot be exploited due to its Overall Equipment Effectiveness (OEE) low values. The aim is to contribute to a better knowledge of the application technique to reduce waste in process industries so that, through an analysis of their production data, the problems that prevent companies from being efficient are identified and, through the selection, development and implementation of the techniques of the Lean Manufacturing Methodology, SME can be oriented to carry out improvement actions in their production systems at a low cost.
\end{abstract}

Keywords: Lean Manufacturing; process industries; Total Productive Maintenance; waste; OEE.

\section{INTRODUCTION}

Due to globalization, manufacturing organizations had to adapt their manufacturing strategies to the changes in the world economy. Increasing competition and rapid strategic positioning of companies in the same industry led organizations to improve, with limited resources, their quality and productivity parameters. Thus, many organizations have realized that their survival in business depends fundamentally on producing high quality goods and services.

This research proves the hypothesis that the use and development of TPM-Lean Manufacturing methodology improves the overall equipment effectiveness (OEE) of production, taking into account the analysis of failures and reliability of the equipment in addition to the forecast of the demand for spare parts in a metalworking company.

Therefore, the contribution of this study is to show how using TPM-Lean Manufacturing methodology increases the OEE by stablishing a relationship between compliance of preventive maintenance and the forecast of future production, and by focusing on improving the quality factor by reducing defects in the manufactured products. The objective of this methodology is summarized in achieving efficient world-class companies with a lower investment.

This article is designed to be used by different companies in the manufacturing sector, especially by small and medium-sized enterprises (SMEs) that need to collect data such as process time per unit and per manufactured products, equipment or machinery maintenance time, and causes that generate defects in manufactured products.

1 Industrial engineer from Universidad Peruana de Ciencias Aplicadas (Lima, Peru). Currently working as head of production at Frecep SAC, a metalworking company (Lima, Peru). ORCID: https://orcid.org/0000-0002-1189-8376

Corresponding author: suministrosproyectoss@gmail.com 
The study is quantitative to reach a deep descriptive and pre-experimental analysis. Descriptive because it focuses on describing the data, processes, or any other element that is subjected to analysis; and pre-experimental because it focuses on proving that the independent variable modifies the dependent variable.

\section{Lean Manufacturing}

Lean Manufacturing appeared as a solution to reduce waste in production processes by implementing the concepts originated in the Toyota Production System (TPS), which were developed by Eiji Toyoda, Taiichi Ohno and Shigeo Shingo in the early 1940s. It has been directly applied to discrete industries, that is, assembly industries. However, applicability to process industries, that is, continuous industries, is still under development. Recently, scholars such as Abdulmalek and Rajgopa (2007); King (2019), Mahapatra and Mohanty (2007); Marodin et al. (2018); and Pool et al. (2011) have been analyzing and implementing some of the Lean concepts in process industries and have achieved remarkable results. The goal of Lean Manufacturing is to reduce waste at every stage of the production process.

For Hu et al. (2015), Lean Manufacturing methodology is increasingly applied in manufacturing companies, regardless of their size, in order to make them more competitive and sustainable and to achieve positioning in the modern market. The application of this methodology also resulted in great agility in production processes. Therefore, the applicability of Lean Management in SMEs is recognized as crucial for the development of economies around the world.

Powell et al. (2009) acknowledge that, although many of the Lean practices and tools have been successfully applied in all types of industrial processes, there is a significant lack of the application of control practices of Lean production in process industries.

Abdulmalek and Rajgopal (2007) present a casebased approach to show how lean manufacturing practices, when used appropriately, help eliminate waste in process industries, keep a better inventory control, improve product quality, and contribute to a better overall financial and operational control. Not all lean tools are applicable in the same manufacturing environments; therefore, it must be identified which lean tools are applicable in each manufacturing environment within the process industries.
There are lean methodologies that are applicable to all types of manufacturing environments in the process sector, regardless of their specific characteristics. These tools offer the possibility to obtain significant profit with relatively low investments since they require simple application (Abdulmalek \& Rajgopal, 2007; Powell et al., 2009).

Manufacturing flexibility is very important for agility and can be improved with an appropriate Lean implementation. It means that industries make what the customer orders as soon as possible after the order has been placed (Saleeshya et al., 2012).

\section{TPM and OEE}

According to Jasiulewicz-Kaczmarek (2016), total productive maintenance (TPM) is a set of strategic initiatives focused on maintaining and improving production and quality systems through machines, equipment, processes, and employees that add value to an organization.

TPM has eight pillars aimed mainly at proactively improving machine reliability. People are at the center of this system and must be continuously trained to identify and eliminate waste (Zarreh et al., 2018).

The publications on TPM agree that this methodology has a participatory work philosophy, whose objective is to maximize the effectiveness of machinery and equipment by eliminating errors, defects and other negative phenomena.

According to Moreira et al. (2018), OEE is a performance indicator that allows measuring the overall effectiveness of the equipment that makes it possible to know the productive efficiency of a company, department or machine.

Nakajima (1988) defines six types of losses and gathers them in three groups, associating each group to an indicator; thus, he mentions that there are: (a) losses due to the unavailability of equipment, (b) losses due to poor equipment functioning or performance, and (c) losses due to manufacturing low-quality products. Nakajima associates these groups to their indicators availability $(A)$, performance $(P)$ and quality $(Q)$ respectively.

The overall equipment effectiveness (OEE) indicator is obtained by multiplying the three indicators previously mentioned. Figure 1 illustrates the procedure to calculate OEE and shows its relationship with the six types of losses mentioned above. Given the emergence of new production schemes with increasingly complex systems, the OEE calculation must also be supported by new technologies in order to guaran- 
tee the quality of the information, de igual manera éste debe estructurarse bajo una buena estrategia de manufactura como lo es la filosofía "Lean" y debe de ser complementado por la metodología de mejora continua [likewise, it must be structured under a good manufacturing strategy such as the Lean philosophy and must be complemented by the continuous improvement methodology] (García et al., 2011).

\section{Failure Analysis}

The Weibull distribution is one of the most used distributions in reliability and maintenance engineering. Depending on the value of the shape parameter, $\beta$ (beta), the failure rate can decrease, be constant or grow. It is used to model the failure behavior of various real-life systems. (Chaurasiya et al., 2017; Bistouni \& Jahanshahi, 2015).

\section{MTBF and MTTR}

According to Saetta, and Caldarelli, (2018), Mean Time Between Failures (MTBF) is a KPI related to the average production time elapsed between a failure and the next time it happens.

$$
\text { MTBF }=\frac{\text { Available Production Time }}{\text { Machine Stops }+ \text { Other Stops }}
$$

They also indicate that the Mean Time To Repair (MTTR) is the time it takes to make a repair after the failure has occurred. In other words, it is the time used during the intervention in a given process.

$$
\text { MTTR }=\frac{\text { Time of Machine Stops }+ \text { Time of Other Stops }}{\text { Machine Stops }+ \text { Other Stops }}
$$

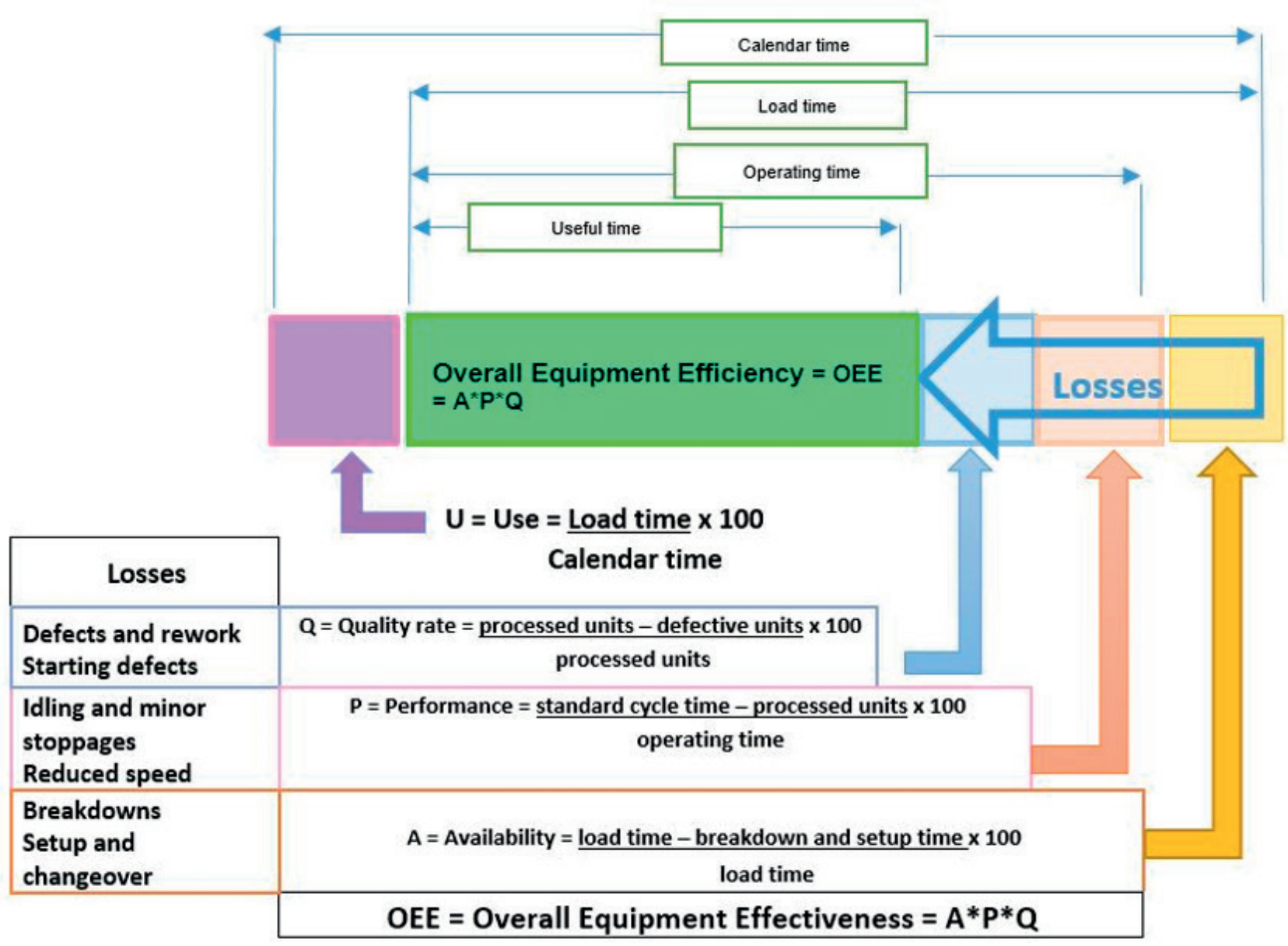

Figure 1. Overall Equipment Effectiveness and its link to the six major losses in production. Source: García et al. (2011). 


\section{METHODOLOGY}

Hernández et al. (2010) state that el investigador puede incluir en su estudio dos o más variables independientes. Cuando en realidad existe una relación causal entre una variable independiente y una dependiente, al variar intencionalmente la primera, la segunda también variará [the researcher can include two or more independent variables in their study. When a causal relationship between an independent variable and a dependent variable does exist, by intentionally varying the first, the second will also vary] (p. 54); for example, if there is a causal relationship between the TPM methodology and OEE, the implementation of TPM will affect OEE, so it can be said that this research is pre-experimental.

According to Tamayo (2003), experimental research can present different types of experimental designs, the best known being the pre-experimental design; therefore, the design of this research is pre-experimental, because it has a pre-test and post-test control group.

Hernández et al. (2010), state that quantitative research usa la recolección de datos para probar hipótesis, con base en la medición numérica y el análisis estadístico, para establecer patrones de comportamiento y probar teorías [uses data collection to prove hypotheses, based on numerical measurement and statistical analysis, to establish behavior patterns and to prove theories] (p. 4).

Tamayo (2003) states that la investigación cuantitativa consiste en el contraste de teorías ya existentes a partir de una serie de hipótesis surgidas de la misma [quantitative research consists of contrasting already existing theories based on a series of hypotheses derived from it]. This author also emphasizes la necesidad de trabajar con una muestra representativa de una población o fenómeno objeto de estudio [the need to work with a representative sample of a population or phenomenon under study].

Cauas (2006) states that utiliza la recolección y el análisis de datos para contestar preguntas de investigación y probar hipótesis establecidas previamente, confía en la medición numérica, el conteo $y$ frecuentemente en el uso de estadísticas para establecer con exactitud, patrones de comportamiento en una población [it uses data collection and analysis to answer research questions and to prove hypotheses previously established, relying on numerical measurement, counting and frequently on the use of statistics to accurately establish behavior patterns in a population] (p. 39).

According to Hernández et al. (2010), descriptive research aims to specify properties, characteristics and important features of any phenomenon analyzed.

For Tamayo (2003), el tipo de investigación descriptiva comprende el registro, análisis e interpretación de la naturaleza actual, y la composición o procesos de los fenómenos [descriptive research comprises the recording, analysis and interpretation of nature, and phenomena composition or processes]. The author advises working on factual realities and their fundamental characteristic of presenting a correct interpretation.

To verify that Lean methodology is applicable to problem solving in an SME process company, the company Frecep SAC, manufacturer of spare parts for mining machinery and equipment, based in Lima, has been selected.

This company presents a large number of finished products delivered with excessive delays to its customers, which causes their dissatisfaction. As a consequence, the company loses more clients, its production costs increase, its profits are reduced, and risks being excluded from the market.

This research is a descriptive and pre-experimental study with a quantitative approach, since it works with the database provided by the company. This database is used to carry out an analysis and measurement of the improvements implemented.

Since Lean Manufacturing is a work philosophy that aims to improve and optimize any production system focusing on the identification and elimination of waste, which are the activities that consume more resources than necessary, the methodology suggested by the different authors consulted in this case study will be applied.

As the main data source, the information of 2018 production provided by the metalworking company was analyzed so that it would allow to select the operational tools that support the applicability of the Lean methodology for this case. Thus, it was detected that the company under study presents low OEE in the spare parts production line, which generates unnecessary costs mainly due to non-compliance with the preventive maintenance program, which in turn generates numerous stops of its machines and increases repair work for corrective maintenance. Consequently, low quality final products were obtained, and the spare parts manufactured were delivered late. 


\section{ANALYSIS}

\section{Unit of Analysis}

The unit of analysis of this research is a part manufactured in the metalworking company under study.

\section{Study Population}

The population chosen for this study was the total spare parts production in 2018 , that is, the 789 parts manufactured, as shown in Table 1.

\section{Sample Size}

For the calculation of the sample size, a population $\mathrm{N}$ of 789 pieces (total production in 2018) has been considered. The value of $Z$ refers to the confidence level, which has been selected at $95 \%$; consequently, the error $(E)$ will be 0.05 . Table 2 shows the sample size calculation.

\section{Sample Selection}

The sample was selected from the historical database of and only those parts manufactured with defects. The sample represents $57.71 \%$ of the population, as shown in Table 3 below.

\section{Data Collection Techniques}

The company database, which was provided by the production control and planning area, was used.

Table 1. Production in 2018.

\begin{tabular}{|lc|}
\hline Production & Frequency \\
\hline Bushing & 38 \\
Shaft & 57 \\
Shaft pinion & 39 \\
Engine shaft & 76 \\
Engine gear & 96 \\
Helical gear & 173 \\
Spur gear & 135 \\
Spur pinion & 40 \\
Transmission system & 115 \\
Engine mount & 20 \\
\hline Total & 789 \\
\hline
\end{tabular}

Source: Prepared by the author.

Table 2. Sample Calculation.

\begin{tabular}{clc}
\hline $\mathrm{n}$ & Sample & $\mathrm{X} ?$ \\
$\mathrm{Z}$ & Confidence level constant & 1.95 \\
& Confidence level & $95 \%$ \\
$\mathrm{p}$ & Individuals possessing the study characteristic & 0.5 \\
$\mathrm{q}$ & $1-\mathrm{p}$ & 0.5 \\
$\mathrm{~N}$ & Population & 789 \\
$\mathrm{e}$ & Sample error & 0.05 \\
$\mathrm{n}=$ & & 247 \\
\hline
\end{tabular}

Source: Prepared by the author.

Table 3. Types of Failures.

\begin{tabular}{|lccc|}
\hline Causes of defective parts & Subcauses & Frequency & Total \\
Poor measuring and cutting & Machine failure & 71 & 122 \\
Deficient turning & Machine failure & 75 & 138 \\
Deficient milling & Machine failure & 45 & 83 \\
Deficient grinding & Machine failure & 56 & 85 \\
Total & & 247 & 428 \\
Equivalent & & $57.71 \%$ & \\
\hline
\end{tabular}

Source: Prepared by the author. 
The production reports, equipment failure reports, and preventive and corrective maintenance records were used to describe the processes and to analyze the variables provided, which belonged to the production line of spare parts for mining equipment of the company. Figure 2 shows the scheme used for data processing and analysis.

By analyzing the information, it has been detected that the operation of the company under study is characterized by the following results:

- Of the total of 789 units produced in 2018 , 340 were delivered late, representing $43.09 \%$ of the annual production.

- Non-compliance with the preventive maintenance program in 2018 was $93.75 \%$, since out of 16 scheduled preventive maintenance jobs, 4 scheduled for each machine (cutter, lathe, milling machine and grinding machine), only 1 was complied with.
- The excess of corrective maintenance hours in 2018 was $96.76 \%$, that is, 239 processes out of the 247 processes selected as a sample, exceeded the hours scheduled for each corrective maintenance.

- Reprocesses and shrinkage were generated as non-conforming products: for the production of 789 units, 414 reprocesses were generated during manufacturing, and 393 products were shrinkage, so it is assumed that 1596 new processes were opened to produce 789 units; the reprocesses represented $25.94 \%$, and the processes that generated shrinkage were equal to $24.62 \%$ and the products delivered were $49.44 \%$ of the 1596 processes.

- The problems occurred due to work performed with deficiencies in the areas of measuring and cutting, turning, milling and grinding. The occurrence of machine failures has a great incidence in these areas (58.2\%

\section{DATA PROCESSING AND ANALYSIS}

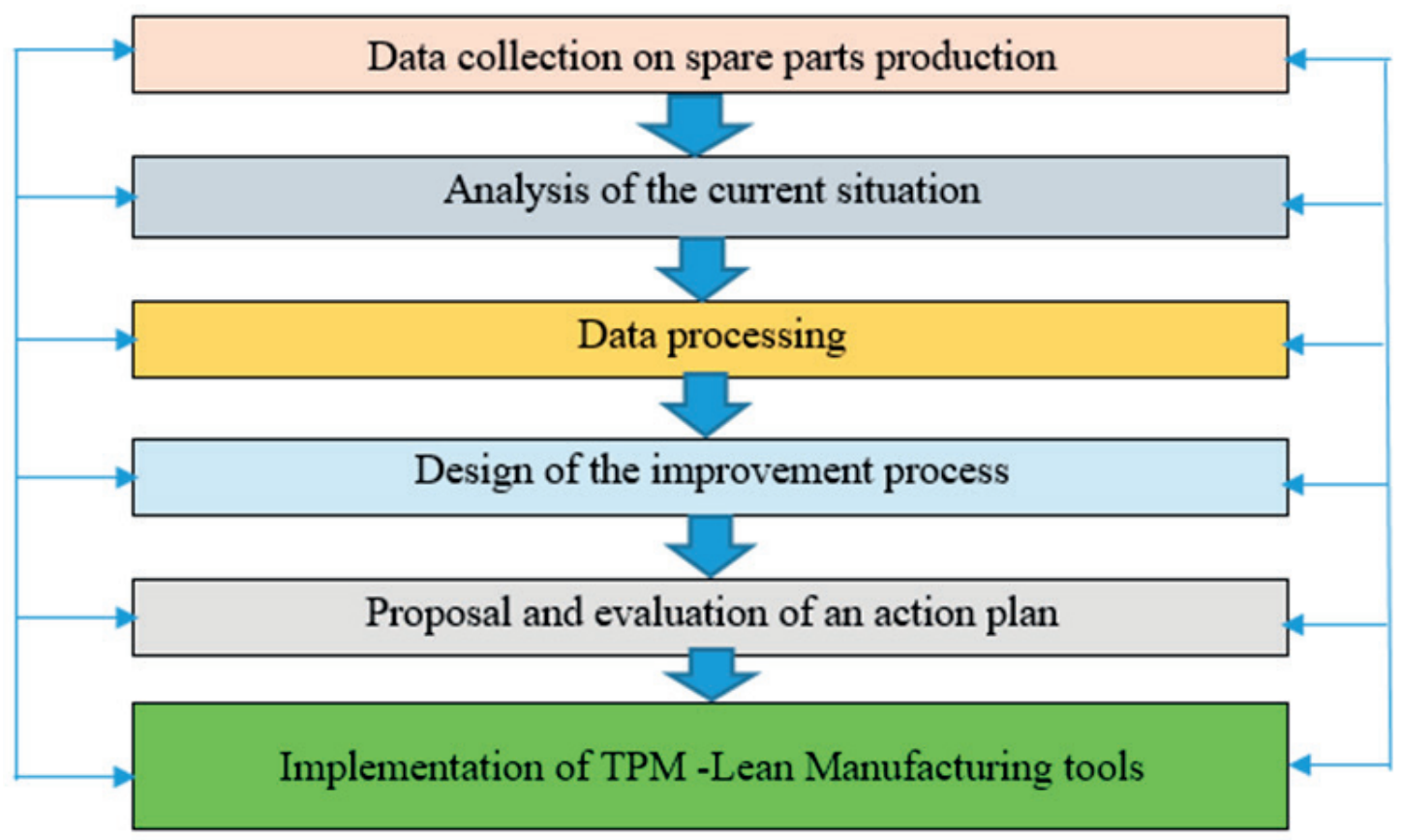

Figure 2. Data processing and analysis scheme. Source: Prepared by the author. 
in measuring and cutting; $54.35 \%$ in turning; $54.22 \%$ in milling and $65.8 \%$ in grinding).

It is inferred that equipment failure is caused by the lack of preventive maintenance work on them, which has generated losses of time, resources, materials and money; also, the use of additional human resources to cover inspections in reprocessing means a great loss in productivity.

Having identified the problem, total productive maintenance and the detection, prevention and elimination of waste were selected as techniques of Lean Manufacturing methodology to be used to improve the performance of the company under study.

\section{Hypothesis Proposition and Testing}

In order to have the data processed for the current study, Microsoft Excel software was used, specifically its statistical tools and those created to build customized tables and graphs. For the interpretation of the statistical results, the books by Chue et al. (2009) and Córdova (2003) were used.

The hypothesis tests of failure analysis, reliability and demand were performed, as well as the general hypothesis test, which, in order to be validated, requires the previous validation of the three previous hypotheses. The results of the general hypothesis test will be shown, since the four mentioned have the same scheme.

\section{General Hypothesis Test}

This hypothesis must be validated by means of the three specific hypotheses, since these are required to accept or reject the general hypothesis by means of the OEE values.

Null hypothesis $-\mathrm{H}_{0}$ : The use of the TPM-Lean Manufacturing methodology does not improve the OEE in the production of spare parts for mining equipment in the company Frecep SAC.

Alternate hypothesis $-\mathrm{H}_{1}$ : The use of the TPM-Lean Manufacturing methodology does improve the OEE in the production of spare parts for mining equipment in the company Frecep SAC.

To validate the hypotheses, a Student's t-test for paired samples will be used, selecting a reliability of $95 \%$. Once the result is obtained, the null hypothesis will be rejected if $t<-2.776$ and if $t>2.776$. The data taken before and after TPM implementation corresponding to current and improved OEE values will be used as shown in Table 4.

Where, t stat $=-20.446$, that is, it is less than -2.776 ; then, the null hypothesis is rejected, and the alternative hypothesis is accepted. Since there is a confidence level of $95 \%$, the alternate general hypothesis is accepted, which states: "The use of the TPM-Lean Manufacturing methodology does improve the OEE in the production of spare parts for mining equipment in the company Frecep SAC".

\section{Application of the TPM Technique.}

For the implementation of TPM, the pillar planned maintenance and the pillar focused improvement were executed, which refers to analyze failures and improve the maintenance management process.

Table 4. Student's T-Test - General Hypothesis.

\begin{tabular}{|lcc|}
\hline \multicolumn{1}{|c|}{ t-Test: Paired Two Sample for Means } & Current & Improved \\
\hline Mean & 133.0000000 & 65.3352210 \\
Variance & 423.0909091 & 4.9223734 \\
Observations & 12 & 12 \\
Pearson's correlation & 0.5808295 & \\
Hypothetical mean difference & 0 & \\
Df & 11 & \\
t Stat & 12.1042398 & \\
P(T< = t) one-tailed & 0.0000001 & \\
t Critical one-tailed & 1.7958848 & \\
P $(T<=$ t) two-tailed & 0.0000001 & \\
t Critical two-tailed & 2.2009852 & \\
\hline
\end{tabular}

Source: Prepared by the author. 
When analyzing the preventive maintenance schedule, it was observed that in 2018 no planned maintenance (PM) was performed on the cutting, lathe and milling equipment. Due to the non-compliance of PM, which was $93.75 \%$, a break in the operation was caused, which originated a series of corrective maintenance (CM). When only 8.47 hours of PM were fulfilled, 1917.33 hours of CM and 1177.64 hours of excess of CM were originated, that is, for each PM performed, approximately 226 CM were executed.

An Excel sheet was generated to determine the $x$-axis and $y$-axis in order to use the regression tool and thus calculate the Weibull failure parameters $\beta$ and $\alpha$, using the Weibull equations.

Likewise, the MTBF and MTTR indicators were calculated: The MTBF value was 50.86 hours, which means that every 50.86 hours a failure occurs in any of the 4 machines, and the average repair time for each CM is 7.76 hours on average. With this data we can calculate the reliability of the equipment as shown in Table 5.

The calculation of the reliability of the line is low, since at 6 hours it is 0.7139 , at 10 hours it is only 0.2668 and at 19 hours after a failure it is 0 .

By applying the pillar focused improvement, a new preventive maintenance schedule is generated, in which it is calculated that, due to the wear of the equipment, preventive maintenance should be performed every 13 days (MTBF/8 hours per day). The work has been considered 365 days, so there is a frequency of $28 \mathrm{PM}$ in a year.
It has been calculated that the probability of execution of a PM is 0.25 , so it is assumed that out of a frequency of 28 PM for each equipment, 7 must be executed, and in the case of autonomous maintenance (AM), 13 must be executed. This means that when a total of 320 maintenance tasks are programmed, between PM and AM, 80 must be executed. The planning of the PM works is complemented with the creation of the forms and logs to be used to record the verifications in the execution of the works.

Based on the current demand (year 2018) a forecast of the future demand with the improved conditions (year 2019) was made. Thus, it has been calculated that the daily demand for products is an average of 3.52 units per day for 2018 and an average of 3.29 units per day for 2019. Considering that the production per product takes 1.52336 days, the production time for the products in 2019 is very similar when using the four machines for manufacturing.

There was an improvement in the reduction of the delivery times of on time finished products, which was a total of 719 units. This means that of the annual production of 742 units, 23 units were delivered late, due to reprocesses (22) and processes that generated waste (20). Thus, the number of operators was reduced from an average of 8 to 6 , as well as the theoretical hours from 17,908 to 12,420 hours (due to the difference in the number of operators and the decrease in maintenance hours); the programmed hours were reduced from 14700 to 10 200 hours per year; and the hours of classes and meetings from 220.50 to 153 hours. The number of

Table 5. Reliability Calculation - Production Line (4 machines).

\begin{tabular}{|l|c|c|c|c|}
\hline \multicolumn{5}{|c|}{ Reliability Calculation - Production Line (4 machines) } \\
\hline \multicolumn{2}{|c|}{} & Hours & Failure probability & Reliability \\
\hline Beta (slope parameter) = & 2.91 & 0 & 0.0000 & 1.0000 \\
\hline Eta (useful life feature) = & 8.72 & 1 & 0.0031 & 0.9969 \\
\hline MTBF (hours) = & 50.86 & 2 & 0.0232 & 0.9768 \\
\hline MTTR (hours) = & 7.76 & 4 & 0.0735 & 0.9265 \\
\hline & & 5 & 0.1615 & 0.8385 \\
\hline & & 6 & 0.2861 & 0.7139 \\
\hline & & 7 & 0.4359 & 0.5641 \\
\hline & & 10 & 0.5919 & 0.4081 \\
\hline
\end{tabular}

Source: Prepared by the author. 
PM hours was increased from 8.47 to 247.90 hours, which reduced CM from 1917.33 to 65.51 hours, eliminating $\mathrm{CM}$ overtime. Production delay was reduced from 2156.49 to 112.37 hours and production time was reduced from 9602.80 to 9028.33 hours. Downtime was reduced from 794.40 to 592.89 hours by adding 40 hours of MA.

Complying with the PM reduced the $\mathrm{CM}$, since due the lack of PM, mechanical failures of the equipment and the need for the $\mathrm{CM}$ occur; therefore, by forecasting the frequency and hours of PM and CM, the following OEE has been calculated:

Consequently, by improving compliance with the MPs and MAs by $30.84 \%$, the quality factor has improved from $49.44 \%$ to $94.64 \%$, the performance factor from $76.68 \%$ to $93.34 \%$, and, therefore, the availability factor has also increased from $86.70 \%$ to $96.88 \%$. The OEE was increased from $32.86 \%$ to $85.58 \%$, exceeding the world class rate of $85 \%$. It was concluded that non-compliance with PMs generates CMs and it results in project delivery delay. The change in MTBF and MTTR rates are also significant, as shown in Table 6.

When calculating the annual MTBF, it is observed that it has increased from 50.86 hours to 237.65 hours and the MTTR has been reduced from 7.76 hours to 0.27 hours.

It is possible to quantify the savings obtained by implementing the TPM methodology, which amount to S/ $590,353.55$ as a result of reducing the number of operators and the hours and delay per CM. In turn, the investment is $S / 119,317.15$ for the increase in $\mathrm{PM}$ and $\mathrm{AM}$ and for the training of operators for proper management of maintenance work. This is shown in Table 7.

\section{RESULTS}

As a final result, it is concluded that by improving compliance with PM (preventive maintenance) and AM (autonomous maintenance), the quality factor has improved (from $49.44 \%$ to $94.64 \%$ ), as well as the performance factor (from $76.68 \%$ to $93.34 \%$ ), and, consequently, the availability factor has increased (from $86.70 \%$ to $96.88 \%$ ); therefore, the OEE increased from $32.86 \%$ to $85.58 \%$, exceeding the worldwide rate of 85 . In addition, the relation of preventive and corrective maintenance also improves from 1:226 to 4:1. Table 8 shows the indicators.

Table 6: OEE Calculation: Current - Improvement.

\begin{tabular}{|c|c|c|c|c|c|c|c|}
\hline \multicolumn{7}{|c|}{ Current OEE } & \multicolumn{4}{c|}{ Improvement OEE } \\
\hline Availability & Performance & Quality & OEE & Availability & Performance & Quality & OEE \\
\hline \multicolumn{3}{|c|}{ World Class Standard } & \multicolumn{3}{|c|}{ World Class Standard } \\
\hline $\mathbf{9 0} \%$ & $95 \%$ & $99 \%$ & $\mathbf{8 5} \%$ & $90 \%$ & $95 \%$ & $99 \%$ & $85 \%$ \\
\hline $66.16 \%$ & $76.41 \%$ & $48.78 \%$ & $24.66 \%$ & $87.60 \%$ & $90.30 \%$ & $94.10 \%$ & $74.43 \%$ \\
\hline $90.43 \%$ & $72.86 \%$ & $47.41 \%$ & $31.24 \%$ & $93.72 \%$ & $97.43 \%$ & $93.84 \%$ & $85.69 \%$ \\
\hline $89.51 \%$ & $79.16 \%$ & $52.50 \%$ & $37.20 \%$ & $85.26 \%$ & $88.29 \%$ & $95.40 \%$ & $71.82 \%$ \\
\hline $83.20 \%$ & $72.65 \%$ & $45.07 \%$ & $27.24 \%$ & $88.50 \%$ & $92.23 \%$ & $94.22 \%$ & $76.90 \%$ \\
\hline $88.32 \%$ & $82.45 \%$ & $54.62 \%$ & $39.77 \%$ & $85.34 \%$ & $88.76 \%$ & $95.43 \%$ & $72.29 \%$ \\
\hline $86.79 \%$ & $81.44 \%$ & $50.40 \%$ & $35.62 \%$ & $91.84 \%$ & $95.62 \%$ & $95.22 \%$ & $83.62 \%$ \\
\hline $84.25 \%$ & $74.57 \%$ & $48.63 \%$ & $30.55 \%$ & $94.00 \%$ & $98.45 \%$ & $93.85 \%$ & $86.85 \%$ \\
\hline $89.62 \%$ & $74.09 \%$ & $49.34 \%$ & $32.76 \%$ & $87.61 \%$ & $91.40 \%$ & $94.11 \%$ & $75.36 \%$ \\
\hline $83.11 \%$ & $75.67 \%$ & $53.49 \%$ & $33.64 \%$ & $93.99 \%$ & $97.87 \%$ & $96.84 \%$ & $89.08 \%$ \\
\hline $87.82 \%$ & $74.51 \%$ & $48.25 \%$ & $31.57 \%$ & $89.84 \%$ & $94.69 \%$ & $93.61 \%$ & $79.64 \%$ \\
\hline $82.23 \%$ & $78.89 \%$ & $48.74 \%$ & $31.62 \%$ & $91.59 \%$ & $95.53 \%$ & $95.22 \%$ & $83.31 \%$ \\
\hline $87.70 \%$ & $77.45 \%$ & $48.97 \%$ & $33.26 \%$ & $86.28 \%$ & $89.49 \%$ & $94.00 \%$ & $72.58 \%$ \\
\hline $86.70 \%$ & $76.68 \%$ & $49.44 \%$ & $32.86 \%$ & $96.88 \%$ & $93.34 \%$ & $94.64 \%$ & $85.58 \%$ \\
\hline
\end{tabular}

Source: Prepared by the author. 
MTBF (mean time between failures) and MTTR (mean time to repair) are also important. When calculating the annual MTBF, it is noted that its value has increased from 50.86 hours to 237.65 hours and the MTTR has been reduced from 7.76 hours to 0.27 hours, as shown in Tables 9 and 10 .

Finally, the maintenance management process has been changed by reducing the activity, as illustrated in Figure 3, assuming that the PMs are met, as established in this research.

\section{CONCLUSIONS}

After having developed this research to demonstrate the feasibility of the application of the TPMLean Manufacturing methodology in manufacturing companies of metal spare parts, the following conclusions have been determined:

- The use and application of the TPM-Lean Manufacturing methodology is convenient to show the diagnosis of the current state of the company and to find and propose alternatives in order to achieve the improvement of a manufacturing process.

- The influence of the Lean Manufacturing tools in the increase of the efficiency of the production of spare parts for equipment of the mining sector in the company FRESEP SAC is tangible.

- By means of the adequate processing of a necessary database, the fundamental causes of the low OEE in the production of spare parts can be determined; these causes, in the case of the company under study, are the non-compliance of preventive maintenance and the excess of hours used in corrective maintenance, as obtained through the use of the TPM methodology.

- Through the application of the TPM methodology, the OEE in the production of spare parts for mining sector equipment in the metalworking company FRESEP SAC has increased from $32.86 \%$ to $85.58 \%$.

- To implement the proposed improvements in the company under study, an investment of S/ 119 317.15 is required, which is justifiable, since it generates savings of S/ 590353.55 .

- The proposed objectives have been achieved and the hypotheses proposed in this research have also been demonstrated, showing that its application is feasible for manufacturing companies in the SME sector at low costs.

\section{REFERENCES}

[1] Abdulmalek, F., \& Rajgopal, J. (2007). Analyzing the benefits of lean manufacturing and value stream mapping via simulation: A process sector case study. International Journal of Production Economics, 107(1), 223236. Retrieved from https://doi.org/10.1016/j. ijpe.2006.09.009

Table 7. Savings and Investment.

\begin{tabular}{|c|c|c|c|}
\hline \multicolumn{4}{|c|}{ Savings } \\
\hline Operators & 2 (op) & $\mathrm{S} / 1750.00$ & S/ 42000.00 \\
\hline $\mathrm{CM}$ & 1851.82 (hrs) & & S/ 260644.32 \\
\hline Delay due to $\mathrm{CM}$ & 2044.12 (hrs) & & S/ 287709.23 \\
\hline Total & & & S/ 590353.55 \\
\hline \multicolumn{4}{|c|}{ Investment } \\
\hline PM & 239.43 & & S/ 80677.55 \\
\hline AM & 40 & & S/ 13478.27 \\
\hline Training & 4 & & S/ 21675.00 \\
\hline Cost per hour & 68 & & \\
\hline Hours per op & 75 & & \\
\hline Extra hours & 8.75 & 2.19 & S/ 3486.33 \\
\hline Total & & & S/ 119317.15 \\
\hline
\end{tabular}

Source: Prepared by the author. 
Table 8. Indicators.

\begin{tabular}{|llllr|}
\hline \multicolumn{1}{r}{ Indicator } & Current & \multicolumn{1}{c}{ Objective } & \multicolumn{1}{c|}{ Global Standard } & Improvement \\
\hline OEE & $32.86 \%$ & Increase & $85 \%$ & $85.58 \%$ \\
Availability & $86.70 \%$ & Increase & $90 \%$ & $96.88 \%$ \\
Performance & $76.68 \%$ & Increase & $95 \%$ & $93.34 \%$ \\
Quality & $49.44 \%$ & Increase & $99 \%$ & $94.64 \%$ \\
& & & Normal 3:1 & 4.1 \\
Relation PM:CM & $1: 226$ & Increase PM and & Restrictive $5: 1$ & $4: 1$ \\
& & reduce CM & More restrictive $6: 1$ & 4.1 \\
\hline
\end{tabular}

Source: Prepared by the author.

Table 9. MTTR and MTBF Comparison of the Current Production Process 2018.

\begin{tabular}{|cccccccccc|}
\hline Year & Month & $\begin{array}{c}\text { Total } \\
\text { production } \\
\text { hours }\end{array}$ & $\begin{array}{c}\text { Failure } \\
\text { hours }\end{array}$ & $\begin{array}{c}\text { Frequency } \\
\text { of failures }\end{array}$ & MTBF & PM & CM & MTTR CM \\
\hline \multirow{1}{*}{2018} & 1478 & 198 & 22 & 58.15 & 0.00 & 198.15 & 9.01 \\
& Jan & 1182 & 113 & 16 & 66.80 & 0.00 & 113.17 & 7.07 \\
& Feb & 1084 & 114 & 14 & 69.27 & 0.00 & 113.70 & 8.12 \\
& Mar & 1281 & 215 & 30 & 35.51 & 0.00 & 215.07 & 7.17 \\
& Apr & 1084 & 127 & 16 & 59.81 & 0.00 & 126.56 & 7.91 \\
& May & 1084 & 143 & 18 & 52.24 & 0.00 & 143.17 & 7.95 \\
& Jun & 1379 & 217 & 28 & 41.49 & 0.00 & 217.17 & 7.76 \\
& Jul & 1379 & 143 & 18 & 68.66 & 0.00 & 143.15 & 7.95 \\
& Aug & 887 & 150 & 18 & 40.93 & 0.00 & 149.69 & 8.32 \\
& Sep & 1281 & 156 & 22 & 51.11 & 0.00 & 155.98 & 7.09 \\
& Oct & 1084 & 184 & 24 & 37.48 & 8.47 & 184.05 & 7.67 \\
& Nov & 1281 & 157 & 21 & 53.48 & 0.00 & 157.48 & 7.50 \\
\hline
\end{tabular}

Source: Prepared by the author.

Table 10. MTTR and MTBF Comparison of the Improved Production Process 2019.

\begin{tabular}{|c|c|c|c|c|c|c|c|c|}
\hline Year & Month & $\begin{array}{l}\text { Total } \\
\text { production } \\
\text { hours }\end{array}$ & $\begin{array}{c}\text { Failure } \\
\text { hours }\end{array}$ & $\begin{array}{l}\text { Frequency } \\
\text { of failures }\end{array}$ & MTBF & PM & CM & MTTR CM \\
\hline \multirow{13}{*}{2019} & Jan & 887 & 7 & 4 & 219.93 & 13.40 & 6.77 & 0.31 \\
\hline & Feb & 788 & 4 & 4 & 196.03 & 20.10 & 3.87 & 0.24 \\
\hline & Mar & 887 & 4 & 3 & 294.21 & 20.10 & 3.88 & 0.28 \\
\hline & Apr & 887 & 7 & 4 & 219.79 & 20.10 & 7.35 & 0.24 \\
\hline & May & 887 & 4 & 3 & 294.06 & 23.45 & 4.32 & 0.27 \\
\hline & Jun & 788 & 5 & 3 & 261.04 & 20.10 & 4.89 & 0.27 \\
\hline & Jul & 788 & 7 & 4 & 195.15 & 20.10 & 7.42 & 0.26 \\
\hline & Aug & 887 & 5 & 4 & 220.40 & 23.45 & 4.89 & 0.27 \\
\hline & Sep & 788 & 5 & 2 & 391.44 & 20.10 & 5.11 & 0.28 \\
\hline & Oct & 788 & 5 & 4 & 195.67 & 26.80 & 5.33 & 0.24 \\
\hline & Nov & 788 & 6 & 3 & 260.57 & 20.10 & 6.29 & 0.26 \\
\hline & Dec & 887 & 5 & 4 & 220.28 & 20.10 & 5.38 & 0.26 \\
\hline & TOTAL & 10047 & 66 & 42 & 237.65 & 247.90 & 65.51 & 0.27 \\
\hline
\end{tabular}

Source: Prepared by the author. 


\section{Current}

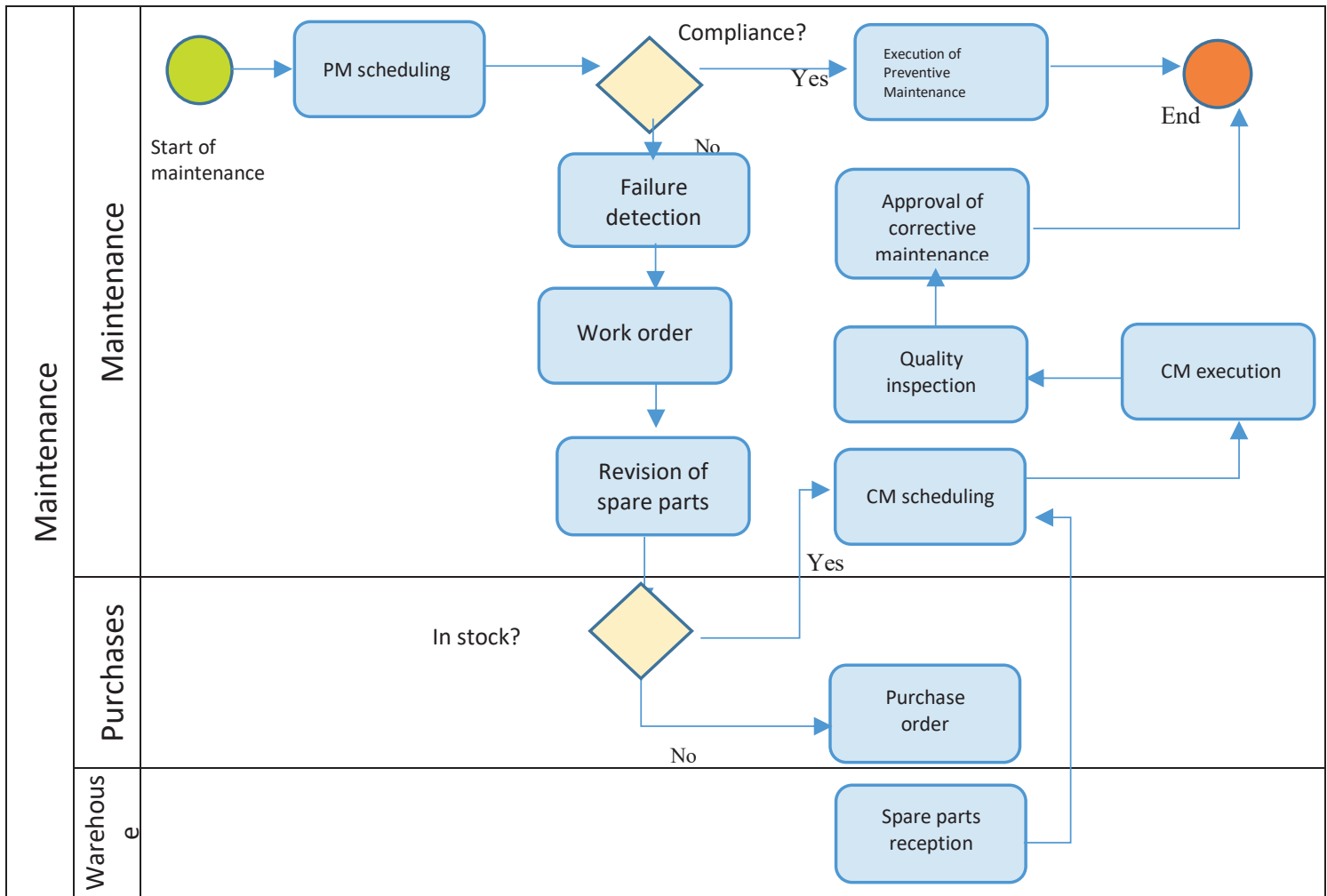

Improvement

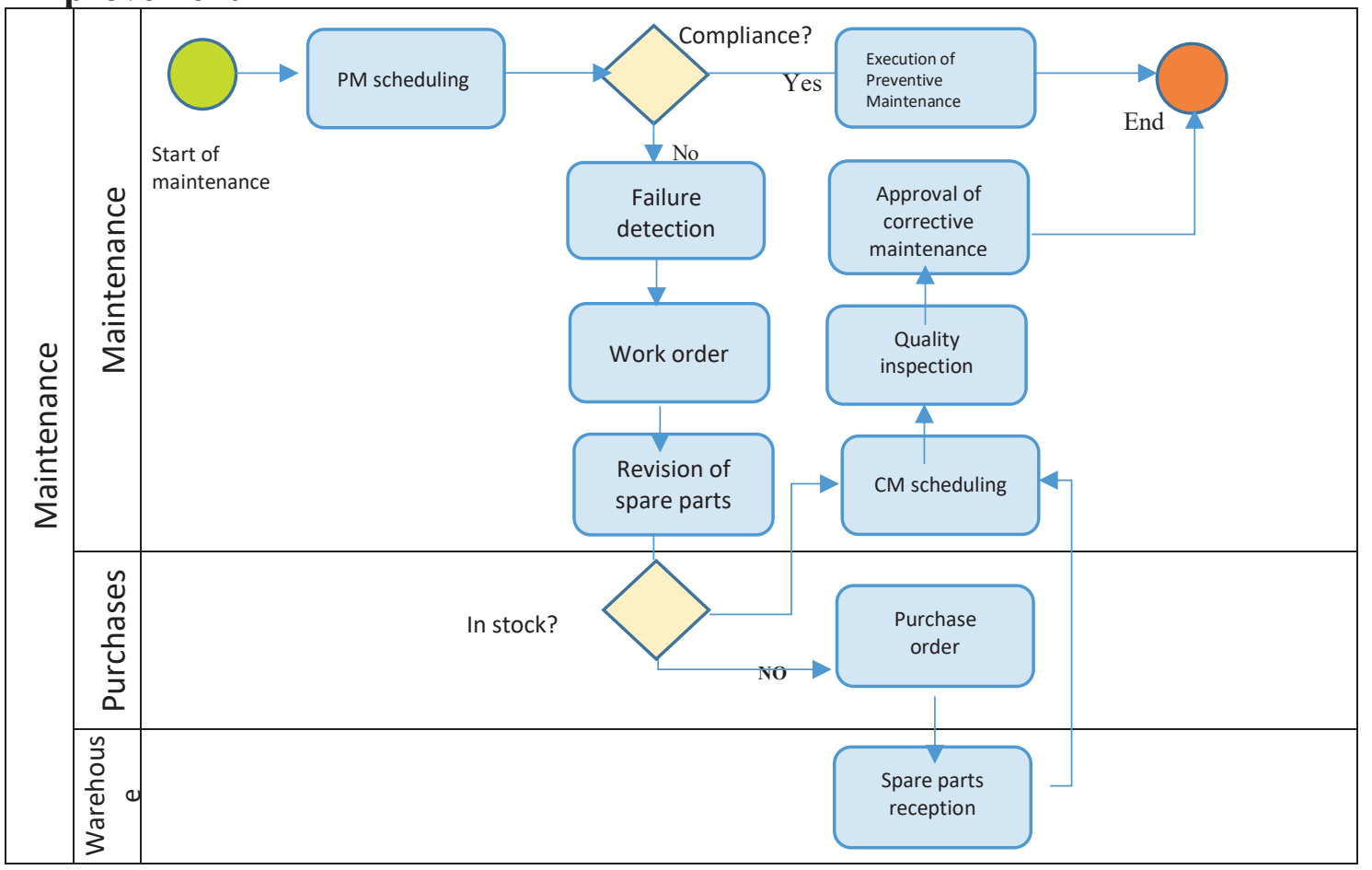

Figure 3. Comparison of Maintenance Management Processes.

Source: Prepared by the author. 
[2] Bistouni, F., \& Jahanshai, M. (2015). Evaluating Failure Rate of Fault-Tolerant Multistage Interconnection Networks Using Weibull Life Distribution. Reliability Engineering \& System Safety, 144, 128-146.

[3] Cauas, D. (2006). Elementos parala elaboración y ejecución de un proyecto de investigación. Investigación en Ciencias Sociales. Retrieved from webjam-upload.s3.amazonaws.com/ apuntesinvest_cauas_1539_.pdf

[4] Chaurasiya, P., Ahmed, S., \& Warudkar, V. (2017). Wind characteristics observation using Doppler-SODAR for wind energy applications. Resource-Efficient Technologies, 3(4), 495505. Retrieved from https://doi.org/10.1016/j. reffit.2017.07.001

[5] Chue, J., Barreno, E., Castillo, C., Millones, R., \& Vásquez, F. (2009). Estadística descriptiva $y$ probabilidades ( $2^{\text {nd }}$ ed.). Lima, Peru: Universidad de Lima.

[6] Córdova, M. (2003). Estadística Descriptiva e inferencial ( $5^{\text {th }}$ ed.). Lima, Peru: Pontificia Universidad Católica del Perú.

[7] García, M., Santos, J., Arcelus, M., \& Viles, E. (2011). Plug\&Lean-OEE game: Juego de entrenamiento basado en el indicador de efectividad global del equipo enfocado en mejorar la productividad de las operaciones de manufactura. Retrieved from https://www. semanticscholar.org/paper/Plug\%26LeanOEE-game\%3A-Juego-de-entrenamientobasado-Garc\%C3\%ADa-Santos/4c2a517417e 320e6ecee6878e892fad4d625031b

[8] Hernández, R., Fernández, C., \& Baptista, P. (2010). Metodología de la investigación $\left(5^{\text {th }}\right.$ ed.). México D.F., Mexico: McGraw-Hill.

[9] Hu, Q., Mason, R., Williams, S., \& Found, P. (2015). Lean implementation within SMEs: a literature review. Journal of Manufacturing Technology Management, 26(7), 980-1012. Retrieved from https://doi.org/10.1108/JMTM02-2014-0013

[10] Jasiulewicz-Kaczmarek, M. (2016). SWOT analysis for Planned Maintenance strategy-a case study. IFAC-PapersOnLine, 49(12), 674679. Retrieved from https://doi.org/10.1016/j. ifacol.2016.07.788

[11] King,P.L.(2019). Leanforthe Process Industries: Dealing with Complexity ( $2^{\text {nd }}$ ed.). New York, United States: Productivity Press. Retrieved from https://doi.org/10.4324/9780429400155
[12] Mahapatra, S., \& Mohanty, S. (2007). Lean Manufacturing in Continuous Process Industry: An Empirical Study. Journal of Scientific \& Industrial Research, 66(1), 1927. Retrieved from http://nopr.niscair.res.in/ handle/123456789/1104

[13] Marodin, G., Frank, A., Tortorella, G., \& Netland, T. (2018). Lean Product Development and Lean Manufacturing: Testing Moderation Effects. International Journal of Production Economics, 203, 301-310. Retrieved from https://doi.org/10.1016/j.ijpe.2018.07.009

[14] Moreira, A., Silva, F., Correia, A., Pereira, T., Ferreira, L., \& de Almeida, F. (2018). Cost reduction and quality improvements in the printing industry. Procedia Manufacturing, 17(1), 623-630. Retrieved from https://doi. org/10.1016/j.promfg.2018.10.107

[15] Nakajima, S. (1988). Introduction to TPM: Total Productive Maintenance. Cambridge, MA, United States: Productivity Press.

[16] Pool, A., Wijngaard, J., \& Van Der Zee, D. (2011). Lean planning in the semi-process industry, a case study. International Journal of Production Economics, 131(1), 194-203. Retrieved from https://doi.org/10.1016/j.ijpe.2010.04.040

[17] Powell, D., Alfnes, E., \& Semini, M. (2010). The Application of Lean Production Control Methods within a Process-Type Industry: The Case of Hydro Automotive Structures. In B. Vallespir, T. Alix (Eds.), IFIP International Conference on Advances in Production Management Systems. Berlin, Germany: Springer, Berlin, Heidelberg. Retrieved from https://doi.org/10.1007/978-3642-16358-6_31

[18] Saetta, S., \& Caldarelli, V. (2018). The machinery performance analysis with smart technologies: a case in the food industry. IFACPapersOnLine, 51(11), 441-446. Retrieved from https://doi.org/10.1016/j.ifacol.2018.08.341

[19] Saleeshya, P. G., Raghuram, P., \&Vamsi, N. (2012). Lean manufacturing practices in textile industries - a case study. International Journal of Collaborative Enterprise, 3(1), 1837. Retrieved from https://doi.org/10.1504/ IJCENT.2012.052367

[20] Tamayo M. (2003). El proceso de la investigación científica ( $4^{\text {th }}$ ed.). México D.F, Mexico: Editorial Limusa, S. A. de C. V. Grupo Noriega Editores.

[21] Zarreh, A., Wan, H., Lee, Y., Saygin, C., \& Al Janahi, R. (2018). Cybersecurity 
Concerns for Total Productive Maintenance in Smart Manufacturing Systems. Procedia Manufacturing, 38(1), 532-539. Retrieved from https://doi.org/10.1016/j.promfg.2020.01.067 